\title{
New southern blue compact dwarf galaxies in the 2dF Galaxy redshift survey $\star, \star \star$
}

\author{
P. Papaderos ${ }^{1}$, N. G. Guseva ${ }^{2}$, Y. I. Izotov ${ }^{2}$, K. G. Noeske ${ }^{1,3}$, T. X. Thuan ${ }^{4}$, and K. J. Fricke \\ ${ }^{1}$ Institut für Astrophysik, Friedrich-Hund-Platz 1, 37077 Göttingen, Germany \\ e-mail: papade@astro.physik.uni-goettingen.de \\ 2 Main Astronomical Observatory, Ukrainian National Academy of Sciences, 27 Zabolotnoho str., Kyiv 03680, Ukraine \\ 3 University of California, 1156 High St., Santa Cruz, CA 95064, USA \\ 4 Astronomy Department, University of Virginia, Charlottesville, VA 22903, USA
}

Received 1 November 2005 / Accepted 12 May 2006

ABSTRACT

\begin{abstract}
Aims. Aiming to find new extremely metal-deficient star-forming galaxies we extracted from the Two-Degree Field Galaxy Redshift Survey (2dFGRS) $100 \mathrm{~K}$ Data Release 14 emission-line galaxies with relatively strong [O III] $\lambda 4363$ emission.

Methods. Spectroscopic and photometric studies of this sample and, in addition, of 7 Tololo and 2 UM galaxies were performed on the basis of observations with the ESO $3.6 \mathrm{~m}$ telescope.

Results. All sample galaxies qualify with respect to their photometric and spectroscopic properties as blue compact dwarf (BCD) galaxies. Additionally, they show a good overlap with a comparison sample of $\sim 100$ well-studied emission-line galaxies on the $12+\log (\mathrm{O} / \mathrm{H})$ vs. $\log (\mathrm{Ne} / \mathrm{O}), \log (\mathrm{Ar} / \mathrm{O})$ and $\log (\mathrm{Fe} / \mathrm{O})$ planes. From the analysis of the $2 \mathrm{dFGRS}$ subsample we report the discovery of two new extremely metal-deficient BCDs with an oxygen abundance $12+\log (\mathrm{O} / \mathrm{H}) \leq 7.6$ and of another seven galaxies with $12+$ $\log (\mathrm{O} / \mathrm{H}) \lesssim 7.8$. Furthermore, we confirm previous oxygen abundance determinations for the BCDs Tol 1304-353, Tol 2146-391, UM 559 and $\mathrm{UM} 570$ to be $12+\log (\mathrm{O} / \mathrm{H}) \leq 7.8$.
\end{abstract}

Key words. galaxies: starburst - galaxies: abundances - galaxies: fundamental parameters - galaxies: structure

\section{Introduction}

The identification of chemically unevolved star-forming galaxies in the local universe, with a nearly pristine chemical composition, is a fundamental quest of contemporary observational cosmology. This is because of several reasons.

First, studies of abundance ratios and abundance gradients in these systems are crucial for our understanding of the early chemical evolution of galaxies and for constraining models of stellar nucleosynthesis and the properties of massive stars in the early universe.

Second, if we wish to understand the spectra of primeval galaxies, it is highly important to understand how the UV radiation field of these systems changes as metallicity decreases. It is well established that the lower the metallicity, the harder is the radiation from massive stars (Campbell et al. 1986). As a consequence, strong high-ionization lines are expected in the spectra of metal-deficient star-forming galaxies. Indeed, deep spectroscopy has led recently to the discovery of high-ionization emission lines, such as [Fe V] $\lambda 4227$ and [Ne V] $\lambda 3346,3426$, in addition to strong He II $\lambda 4686$ emission, in a few of the most metal-poor star-forming galaxies known in the local universe (Fricke et al. 2001; Izotov et al. 2001, 2004a; Thuan \& Izotov 2005).

Third, systematic studies of star-forming galaxies all the way down to a strongly subsolar metallicity are indispensable for

\footnotetext{
* Based on observations collected at the European Southern Observatory, Chile, ESO program 71.B-0032.

$\star \star$ Tables 3-5 are only available in electronic form at http://www.edpsciences.org
}

putting tight observational constraints to the primordial helium abundance, which constitutes one of the key tests of Standard Big Bang Nucleosynthesis. They allow to infer the ratio $\mathrm{d} Y / \mathrm{d} Z$ of the mass fraction $Y$ of helium released into the interstellar medium by stars relative to the mass fraction $Z$ of heavy elements. This quantity determines the slope of the linear regression of ${ }^{4} \mathrm{He}$ against $\mathrm{O}$ and $\mathrm{N}$ and hence directly affects the extrapolation of those regression lines to zero metallicity to determine the primordial helium abundance (Peimpert \& Torres-Peimpert 1974, 1976; Pagel et al. 1992; Izotov \& Thuan 1998, 2004a, and references therein).

Fourth, recent work lends strong observational support to the idea that some among the most metal-deficient star-forming galaxies known in the local universe have formed most of their stellar mass within the last $1 \mathrm{Gyr}$, hence they qualify as young galaxy candidates (Papaderos et al. 2002; Guseva et al. 2003; Izotov \& Thuan 2004b; Pustilnik et al. 2004). Evidently, such systems are excellent nearby laboratories for studying at high spatial resolution the early chemical and dynamical evolution of galaxies and can yield fundamental insight into the process of galaxy formation in the distant universe. In this regard, dedicated searches for metal-poor star-forming galaxies at low redshifts are of great interest.

Blue compact dwarf (BCD) galaxies constitute ideal nearby laboratories for studying active star formation and galaxy evolution under conditions which in many aspects may resemble those in distant low-mass protogalaxies. These systems are gasrich, low-luminosity $\left(M_{B} \gtrsim-18\right)$ objects undergoing an intense burst of star formation in a very compact region $(\leq 1 \mathrm{kpc})$. Other than originally conjectured (Sargent \& Searle 1970), the 
majority of BCDs are not primordial systems, but evolved dwarf galaxies where starburst activity is immersed within an old extended stellar host galaxy (Loose \& Thuan 1986; Kunth et al. 1988; Papaderos et al. 1996a).

With an oxygen abundance $12+\log (\mathrm{O} / \mathrm{H})$ ranging between 7.1 and $\sim 8.4$ BCDs are the most metal-deficient starforming galaxies known in the universe. They are thus excellent laboratories for studying star formation and other physical processes in nearly primordial conditions, similar to those at the time of galaxy formation. While all BCDs show subsolar chemical abundances, it is notoriously difficult to find extremely metaldeficient $(12+\log (\mathrm{O} / \mathrm{H}) \lesssim 7.6)$ objects. The BCD abundance distribution peaks at $12+\log (\mathrm{O} / \mathrm{H}) \approx 8$ with a sharp drop-off at lower values.

One of the first BCDs discovered, I Zw 18 (Sargent \& Searle 1970) with $12+\log (\mathrm{O} / \mathrm{H})$ of $7.17 \pm 0.01$ in its northwest component and of $7.22 \pm 0.02$ in its southeast component (Thuan \& Izotov 2005) continued to hold the record as the most metal-deficient BCD known for more than three decades. Only very recently, has this system displaced in the metallicity ranking by the BCD SBS $0335-052 \mathrm{~W}$ with an oxygen abundance $12+\log (\mathrm{O} / \mathrm{H})=7.12 \pm 0.03$ (Izotov et al. 2005).

So far, less than a couple of ten BCDs with an oxygen abundance $12+\log (\mathrm{O} / \mathrm{H}) \leq 7.6$ have been discovered despite the existence of a extensive amount of data from large CCD sky surveys performed with modern telescopes. For instance, out of $~ 50000$ Sloan Digital Sky Survey (SDSS) galaxy spectra Stasińska \& Izotov (2003) and Izotov et al. (2004b) were able to extract only 310 galaxies with detected [O III] $\lambda 4363$ emission, allowing for a reliable oxygen abundance determination. This sample does not contain any extremely metal-deficient system, with $12+\log (\mathrm{O} / \mathrm{H}) \leq 7.6$. More recently, Izotov et al. (2006) based on a larger sample of 500000 galaxies from the SDSS Data Release 3 also did not find galaxies with $12+\log (\mathrm{O} / \mathrm{H}) \leq 7.6$.

Continuing our search for nearby (redshift $z \lesssim 0.1$ ) metalpoor star-forming galaxies we focus here on a sample of candidate objects selected from the Two-Degree Field Galaxy Redshift Survey (2dFGRS Colless et al. 2001). By visual inspection of the 2dFGRS $100 \mathrm{~K}$ Data Release, we have extracted $\sim 250$ galaxy spectra with strong emission lines and blue continua. Out of this sample we have chosen 14 most promising metal-poor galaxy candidates with strong [O III] $\lambda 4363$ emission for follow-up observations. This is because the latter auroral line is easily detectable in high-temperature and hence lowmetallicity objects. Furthermore, this line can be used as a temperature diagnostic, allowing for a direct determination of the physical conditions and chemical composition of the ionized interstellar medium in galaxies. This criterion also picks out AGNs, which we have rejected by visual examination of each spectrum based on the specific relative intensities of some emission lines (e.g., strong [O II] $\lambda 3727$ and [N II] $\lambda 6583$ emission lines in combination with strong [O III] $\lambda 5007$ emission line, strong He II $\lambda 4686$ and [O I] $\lambda 6300$, etc.).

In this paper we present spectroscopic and photometric follow-up observations of the 14 selected 2dFGRS objects. In addition, we include in our study 7 Tololo and 2 University of Michigan (UM) Survey emission-line galaxies (Salzer et al. 1989a,b; Terlevich et al. 1991). The latter objects were included with the purpose of a) improving their abundance determinations and b) enhancing the efficiency of our observing programme, filling the gap in right ascension between $13^{\mathrm{h}}$ and $22^{\mathrm{h}}$ which was not covered by the galaxies from the $2 \mathrm{dFGRS}$.
This paper is organized as follows: the data acquisition and reduction are described in Sect. 2. Our results, based on the spectroscopic and photometric analysis, are presented in Sects. 3 and 4. We summarize our conclusions in Sect. 5.

\section{Observations and data reduction}

\subsection{Spectroscopy}

Long slit spectra of the sample galaxies in the wavelength range $\lambda \lambda 3400-7400$ were obtained with EFOSC2 (ESO Faint Object Spectrograph and Camera) mounted at the $3.6 \mathrm{~m}$ ESO telescope at La Silla. The programme was carried out in two separate 3-night observing runs, during the periods April 23-25, 2003 and September 30-October 2, 2003. The observing conditions were photometric during two nights of the first run (April 24 and 25 ) but were subject to large sky transparency variations for the remaining nights.

The journal of observations is given in Table 1 with the target galaxies listed in order of increasing right ascension. All observations were performed with the same instrumental setup. We used grism \#11 ( $\lambda \lambda 3400-7400)$ with grating $300 \mathrm{gr} / \mathrm{mm}$ and no second-order blocking filter. The long slit with a width of 1 " was centered on the brightest $\mathrm{H}$ II region of each galaxy. The spectra were binned along the spatial and dispersion axes resulting in a spatial scale along the slit of $0.314 \mathrm{pixel}^{-1}$, and a spectral resolution of $\sim 13.2 \AA$ (FWHM).

Our sample galaxies were mostly observed at low airmass $(<1.2$, cf. Table 1). Spectroscopic observations at a higher airmass were carried out along the parallactic angle. Thus, no corrections for atmospheric refraction have been applied. The total exposure time of typically $1 \mathrm{~h}$ per target was split up into several subexposures (2-6) to allow for a more efficient cosmic ray rejection. Spectrophotometric standard stars were observed for flux calibration. The seeing varied between $\sim 0.9$ and $\sim 1^{\prime \prime}$. 6 . In Fig. 1 we show the slit orientation for each object superimposed on a broad-band CCD $V$ image taken during the same run.

The data reduction was carried out with the $\mathrm{IRAF}^{1}$ software package. This includes bias-subtraction, flat-field correction, cosmic-ray removal, wavelength calibration, night sky background subtraction, correction for atmospheric extinction and absolute flux calibration of the two-dimensional spectrum.

Redshift-corrected one-dimensional spectra of the brightest $\mathrm{H}$ II region in each galaxy (labeled a in Fig. 1) are shown in Fig. 2. Additionally, we extracted spectra for several of the fainter H II regions labeled in Fig. 1. A summary of the spectroscopic properties of the $\mathrm{H}$ II regions is given in Table 2.

\subsection{Photometry}

All but one of the galaxies (Tol 1400-411) included in the spring 2003 sample were observed in the $V$ band $(5 \mathrm{~min})$, whereas for the remaining galaxies both $V$ and $I$ exposures ( 5 min each) were taken. In either observing run we used EFOSC2 in its imaging mode and with a $2 \times 2$ pixel binning, yielding an instrumental scale of 0.314 pixel $^{-1}$.

The available exposures allow to derive surface brightness profiles (SBPs) down to typically $\mu=26 \mathrm{mag} / \square^{\prime \prime}$ in $V$, i.e. they are sufficiently deep for detecting the low-surface

1 IRAF is the Image Reduction and Analysis Facility distributed by the National Optical Astronomy Observatory, which is operated by the Association of Universities for Research in Astronomy (AURA) under cooperative agreement with the National Science Foundation (NSF). 
Table 1. List of observed galaxies.

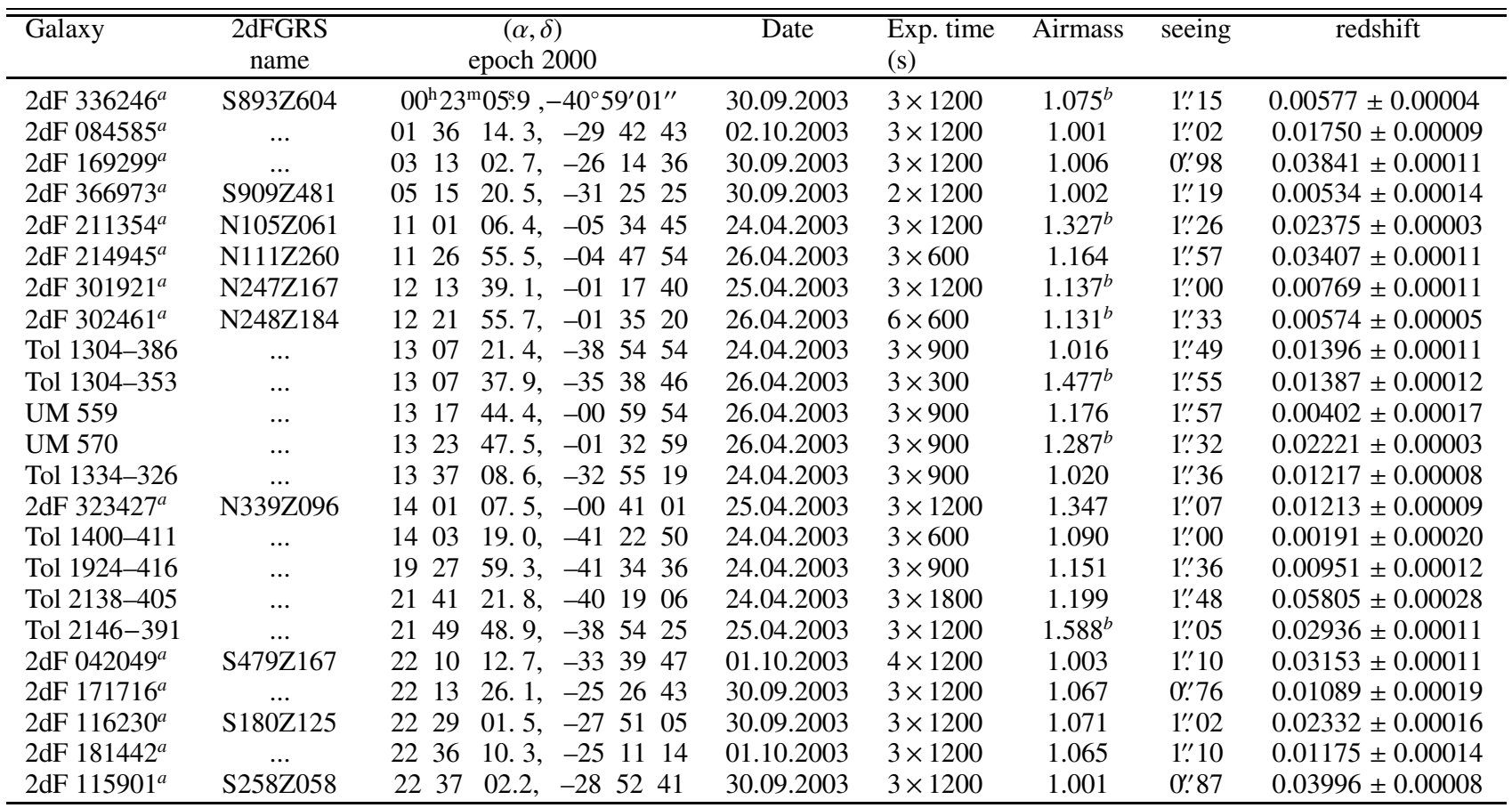

${ }^{a}$ Internal number from the $2 \mathrm{dF}$ Galaxy Redshift Survey $100 \mathrm{~K}$ Data Release.

${ }^{b}$ Spectra taken along the parallactic angle.

brightness (LSB) component in the sample galaxies. Standard imaging reduction procedures were carried out using ESOMIDAS $^{2}$.

A reliable calibration using Landolt (1992) standards could only be established for two nights, April 24 and 25, 2003. A rough calibration is probably also possible for the first half of the night on September 30, 2003. For objects observed in the remaining nights the calibration had to rely by necessity on data from the literature. Part of these images was calibrated using total $B$ and $R$ magnitudes available from the SuperCOSMOS sky survey $^{3}$. The accuracy of these magnitudes may vary from case to case and can be subject to systematic effects which are estimated to yield uncertainties of up to a few tenths of a magnitude. Additional uncertainties are introduced by the conversion of $B$ and $R$ magnitudes to $V$ magnitudes for which we assumed throughout $(B-V) \approx 0.6 \times(B-R)$. This approximative relation was derived by fitting evolutionary synthesis models computed with the PEGASE 2.0 code (Fioc \& Rocca-Volmerange 1997) and refers to a stellar population with an age between $120 \mathrm{Myr}$ and 10 Gyr forming with an exponentially decreasing star formation rate with an e-folding time of $1 \mathrm{Gyr}$. In those models we furthermore assumed a Salpeter initial mass function and a fixed stellar metallicity of $Z_{\odot} / 5$. We did not take into account the effect of nebular line emission on broad band colours, as for the star formation history and the age interval considered here it has a negligible effect on the integral colors.

Note that an integral $(B-V) /(B-R)$ colour ratio of $\approx 0.6$ is also conform with model predictions for a present-day Sd galaxy (see, e.g., Bicker et al. 2004, for details). An approximative $(B-V) /(B-R)$ ratio of $\approx 0.6$ is also consistent with observations

\footnotetext{
${ }^{2}$ ESO-MIDAS is the acronym for the European Southern Observatory Munich Image Data Analysis System which is developed and maintained by the European Southern Observatory.

3 Available from http://www-wfau.roe.ac.uk/sss/
}

of emission-line galaxies (see, e.g., Salzer et al. 1989a, and references therein) and approximates satisfactorily the integral colours of BCDs. For instance, the mean $B-V$ and $V-R$ LSB colours inferred from the BCD sample of Cairós et al. (2001b) are $0.45 \mathrm{mag}$ and $0.33 \mathrm{mag}$, respectively, yielding a $B-V / B-R$ ratio of $\approx 0.6$. The same colour ratio is obtained from the sample of Virgo dwarf ellipticals with available $B V R$ photometry, studied in van Zee et al. (2004).

For Tol 1334-326 and Tol 1304-386 for which SuperCOSMOS data are not available, the $V$ magnitude has been estimated from the $B$ and $R$ magnitudes given in Gil de Paz et al. (2003) and Lauberts \& Valentijn (1989), respectively. Likewise, we have estimated the $V$ magnitude of 2dF 181442 from the $B$ magnitude of Maddox et al. (1990), assuming a mean $B-V$ of 0.4 mag. As no $I$ band magnitudes were available in the literature for the latter system and for $2 \mathrm{dF} 116230$, we have vertically shifted their $I$ band SBPs such as to match the central intensity of $V$ band SBPs. This allows us to quantify the colour variation between the star-forming component and the LSB host galaxy. Finally, the $V$ band exposure of Tol 1924-416 was calibrated using archival HST WFPC2 data (Proposal ID 6708, PI: Östlin) in $V$ and $I$ (filters F555W and F814W, respectively).

Surface photometry has been carried out for all sample galaxies except for $2 \mathrm{dF} 042049$ and Tol 1400-411. The first system is too compact for its LSB host to be studied quantitatively and the second one could not be observed due to scheduling constraints. For a detailed discussion of the latter system the reader is referred, however, to Noeske et al. (2003) who investigated its photometric structure using deep near infrared $J H K^{\prime}$ data. Surface brightness profiles (Fig. 6) were derived using method iv described in Papaderos et al. (2002) and decomposed into the star-forming and LSB components following the procedure detailed in Noeske et al. (2003). 

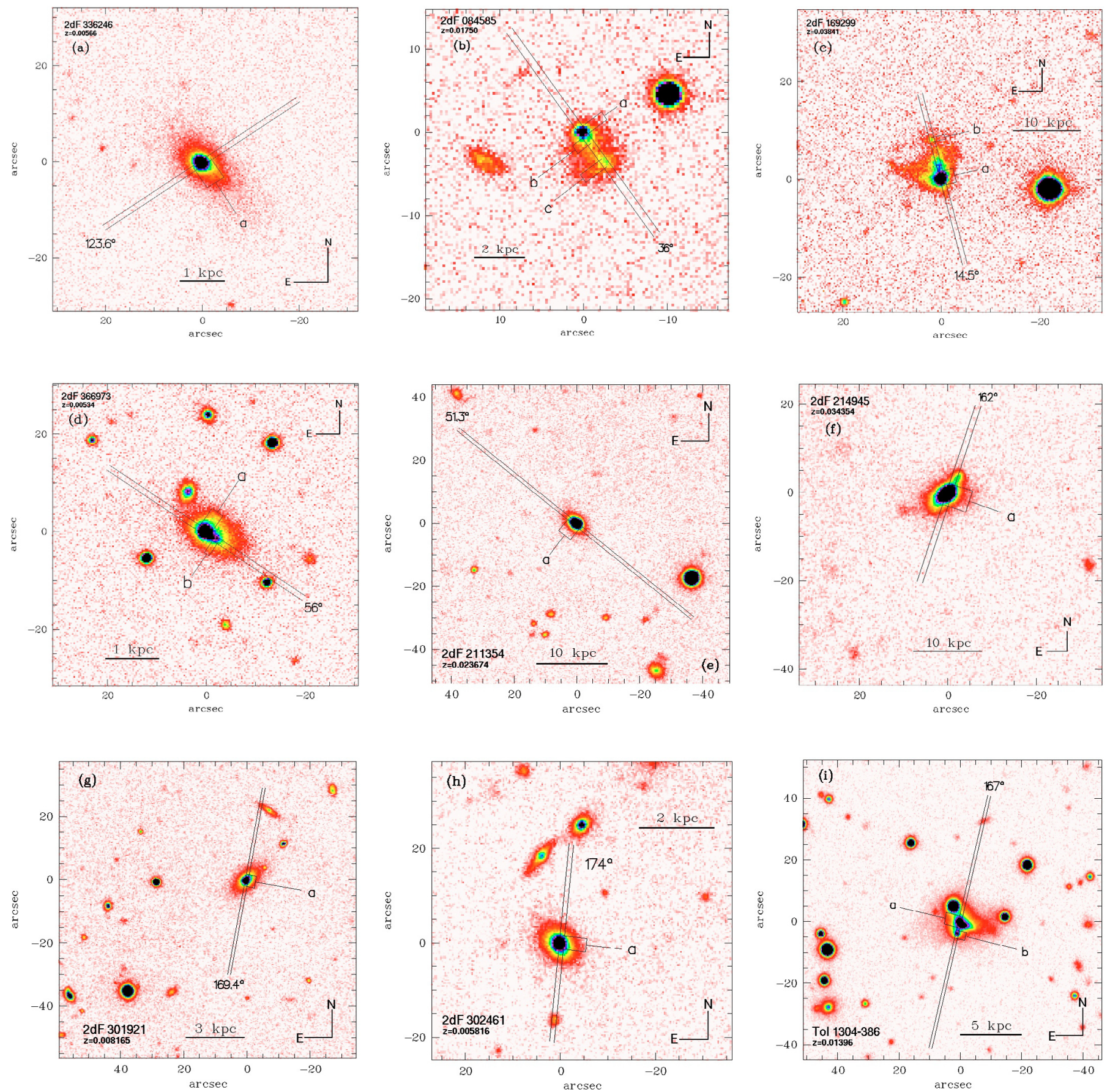

Fig. 1. Reproduction of the slit position on $V$ band EFOSC2 images of our sample galaxies. For Tol 1400-411 (panel o)) for which no $V$ image was acquired we use instead a $J$ band exposure from Noeske et al. (2003). Selected H II regions from which 1D spectra were extracted are indicated. Note that the faint arc-like features in panels $\mathbf{j}$ ) (Tol 1304-353) and $\mathbf{m})$ (Tol 1334-326) are artefacts due to scattered light from nearby bright stars. In all panels north is up and east to the left.

Table 3 lists photometric quantities for 21 galaxies in our sample. All tabulated values are corrected for Galactic absorption based on the extinction maps by Schlegel et al. (1989) available in $\mathrm{NED}^{4}$. In the first column of Table 3 we list the name and morphological type of each target according to the BCD classification scheme of Loose \& Thuan (1986). Column 2 gives the extrapolated central surface brightness $\mu_{\mathrm{E}, 0}\left(\mathrm{mag} / \square^{\prime \prime}\right)$ and exponential scale length $\alpha(\mathrm{kpc})$ of the LSB host galaxy, obtained by fitting an exponential model to the outer part of each SBP. In

\footnotetext{
${ }^{4}$ NASA/IPAC Extragalactic Database; http://nedwww.ipac.caltech.edu
}

Col. 3 we tabulate the apparent $m_{\mathrm{LSB}}$ and absolute $M_{\mathrm{LSB}}$ magnitude of the LSB component in $V$, as derived from the exponential fits. The apparent and absolute $V$-band magnitude, computed from SBP integration are given in Col. 4, and Col. 5 lists the effective radius $r_{\text {eff }}(\mathrm{kpc})$ and mean surface brightness $\mu_{\mathrm{eff}}$ inside

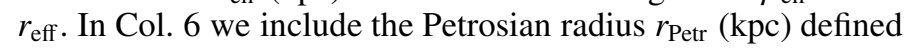
as the radius where the Petrosian $\eta$ function (Petrosian 1976) decreases to a value of $1 / 3$ (see, e.g., Takamiya 1999) and the apparent magnitude $m_{\text {Petr }}$ within that radius. Column 7 (first row) lists a light concentration index based on the radii $r_{80}$ and $r_{20}$ enclosing, respectively, $80 \%$ and $20 \%$ of the total $V$ emission. 

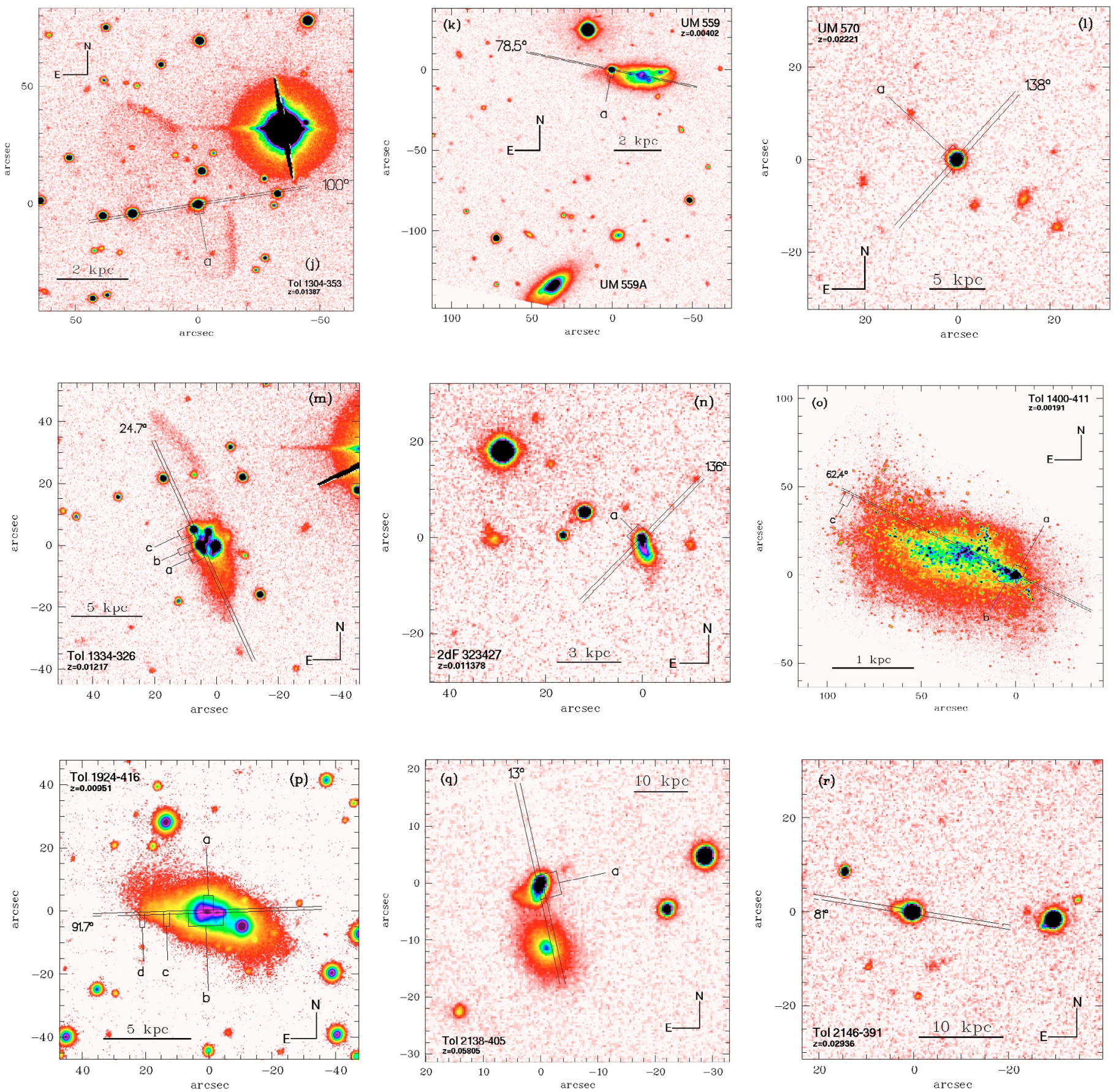

Fig. 1. continued.

In addition, in Col. 7 we tabulate the shape parameter $1 / \eta$ of the Sérsic model which approximates best the observed $V$ SBP. The ratio $r_{25} / r_{\text {eff }}$ of the isophotal radius at $25 \mathrm{Vmag} / \square^{\prime \prime}$ and the effective radius are listed in Col. 8. In the same column we indicate the radius interval in which exponential fits to the LSB emission were applied. The fractional contribution of the LSB component to the total $V$ light and, when available, the mean $V-I$ colour within the fitted radius range are tabulated in Col. 9. In the last column of Table 3 we include the adopted distance in $\mathrm{Mpc}$ and the $V$ band Galactic absorption in mag. Note that distances have been derived after correction of the measured redshifts (Table 1) for the motion relative to the Local Group centroid and the Virgocentric flow (Kraan-Korteweg 1986), assuming a Hubble constant of $75 \mathrm{~km} \mathrm{~s}^{-1} \mathrm{Mpc}^{-1}$.

\section{Spectroscopic properties}

\subsection{Chemical abundances}

The spectra of the brightest star-forming regions in our sample galaxies are characterized by strong nebular emission lines which are superposed on a blue stellar continuum (Fig. 2). Twelve out of the 23 studied systems (those classified nE BCD) possess a single dominant $\mathrm{H}$ II region, while in the remaining ones at least a second H II region can be distinguished. In Fig. 2 we include only the spectrum of the brightest $\mathrm{H}$ II region in each galaxy (region labeled a in Fig. 1).

Emission line fluxes were measured using Gaussian profile fitting. The errors of the line fluxes were calculated from 

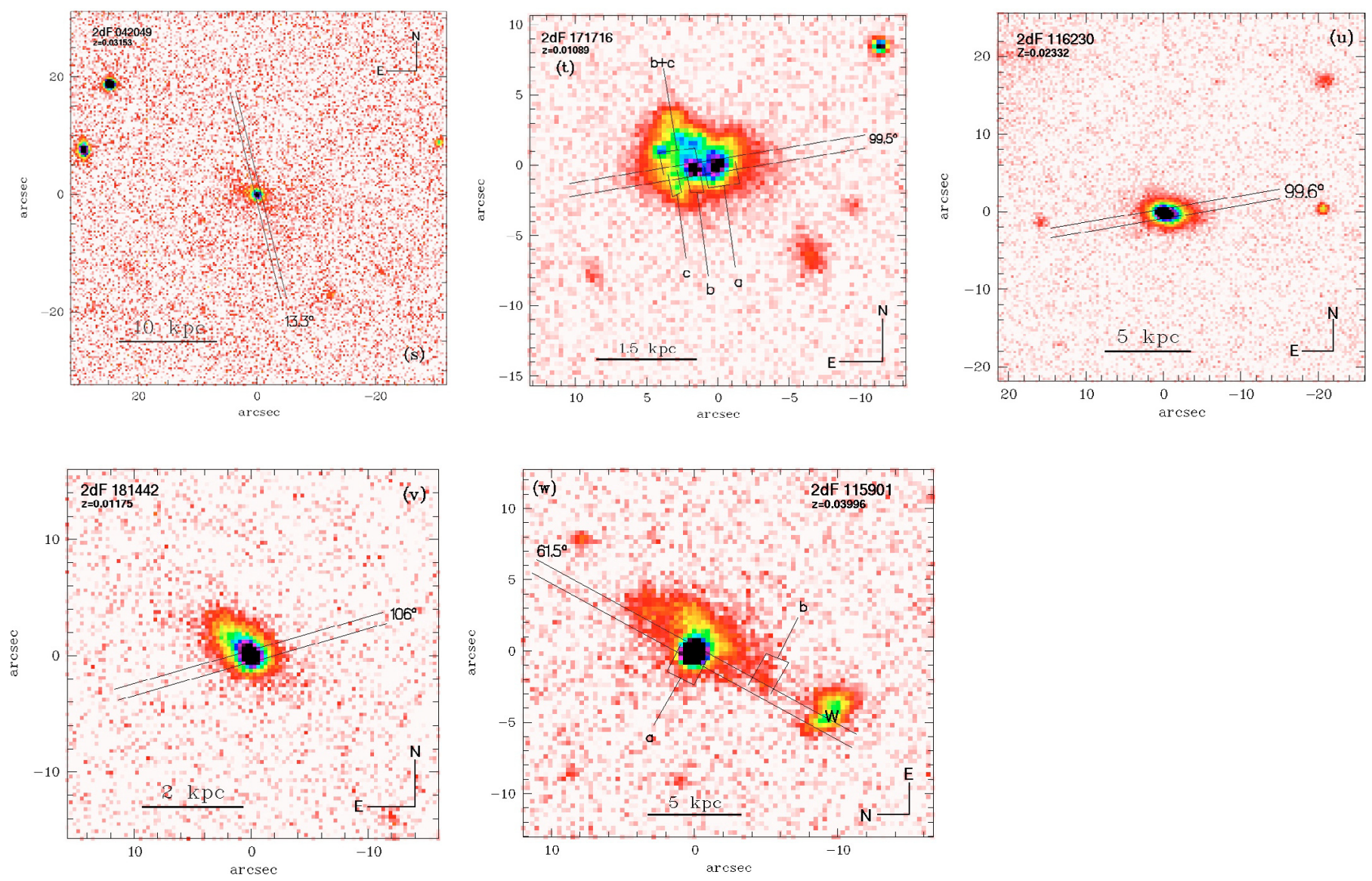

Fig. 1. continued.

the photon statistics in the non-flux-calibrated spectra. These uncertainties have been propagated in the calculations of the elemental abundance errors. Redshifts were derived using the observed wavelength of $\sim 10$ to $\sim 20$ strong emission lines in each spectrum. The redshift errors (Table 1) are calculated as the square root mean deviation of determinations from individual lines from the mean redshift. The observed emission line fluxes $F(\lambda)$ relative to the $\mathrm{H} \beta$ fluxes, equivalent widths $(E W \mathrm{~s})$ of emission lines, extinction coefficients $C(\mathrm{H} \beta)$, observed $\mathrm{H} \beta$ fluxes, and $E W \mathrm{~s}$ of the hydrogen absorption lines are listed in Table 4 for the brightest $\mathrm{H}$ II region of each galaxy.

The electron temperature $T_{\mathrm{e}}$, ionic and total heavy element abundances were derived following Izotov et al. (1994, 1997a) and Thuan et al. (1995). Because of the low spectral resolution of our data the [S II] $\lambda 6717$ and $\lambda 6731$ lines are blended. Therefore, we adopted throughout for the electron number density $N_{\mathrm{e}}(\mathrm{S}$ II $)$ a value of $100 \mathrm{~cm}^{-3}$. Note that the precise value of the electron number density makes little difference in the derived abundances since in the low-density limit which holds for the $\mathrm{H}$ II regions considered here, the element abundances do not depend sensitively on $N_{\mathrm{e}}$. The electron temperatures $T_{\mathrm{e}}(\mathrm{O}$ III $)$ and $T_{\mathrm{e}}(\mathrm{O}$ II) for the high- and low-ionization zones in $\mathrm{H}$ II regions, respectively, ionization correction factors (ICF), ionic and total heavy element abundances for oxygen, neon, argon and, when possible, for iron, in the brightest H II regions (labeled " $\mathrm{a}$ " in Fig. 1) are included in Table 5. Because the [N II] $\lambda 6584$ line is blended with $\mathrm{H} \alpha$ the nitrogen abundance could not be determined with sufficient accuracy using a two-gaussian deblending procedure. Therefore, we prefer not to include it in Table 5. Table 2 contains a synoptic list of the spectroscopic properties of each $\mathrm{H}$ II region selected along the slit in Fig. 1.
In Fig. 3 we plot the $\log (\mathrm{Ne} / \mathrm{O}), \log (\mathrm{Ar} / \mathrm{O})$ and $\log (\mathrm{Fe} / \mathrm{O})$ abundance ratios vs. oxygen abundance for the present and comparison samples. 2dFGRS galaxies are shown by large filled circles and Tololo and UM galaxies by open circles. We also include as filled squares Tol 1214-277 and Pox 186, both observed in the present runs but discussed in the separate papers by Izotov et al. (2004a) and Guseva et al. (2004), respectively.

For comparison, we show by dots in Fig. 3 a sample of $\sim 100$ well-studied low-metallicity BCDs from Izotov et al. (2004b). All spectra in the present and comparison sample were reduced in a homogeneous manner according to the Izotov et al. (1994, 1997a) prescriptions. We adopted the solar abundances from Asplund et al. (2005) (double large open circles in Fig. 3). The best determined ratio, $\mathrm{Ne} / \mathrm{O}$, increases slightly with increasing $\mathrm{O} / \mathrm{H}$ (by $\sim 0.1 \mathrm{dex}$ in the considered metallicity range) which, following Izotov et al. (2006), could be explained by a stronger depletion of oxygen onto dust grains in higher-metallicity galaxies. At the same time, the $\mathrm{Fe} / \mathrm{O}$ abundance ratio shows an underabundance of iron relative to oxygen as compared to solar, suggesting depletion of iron onto dust grains which is especially large for the high-metallicity galaxies. On the other hand, no evident depletion effects are present in the $\mathrm{Ar} / \mathrm{O}$ abundance ratio vs. O abundance diagram. This is because a) the depletion of oxygen is small b) Ar is noble gas and is not locked in grains and c) the scatter of the Ar/O ratio is much larger than that of the $\mathrm{Ne} / \mathrm{O}$ ratio and masks a possible weak trend with the oxygen abundance.

Overall, we see in Fig. 3 a good overlap of our sample galaxies with the comparison sample. An exception is $2 \mathrm{dF} 171716$ (labeled $171716 a$ ) which shows a conspicuously large $\log (\mathrm{Ne} / \mathrm{O})$ ratio, presumably because of a large error caused by the low 


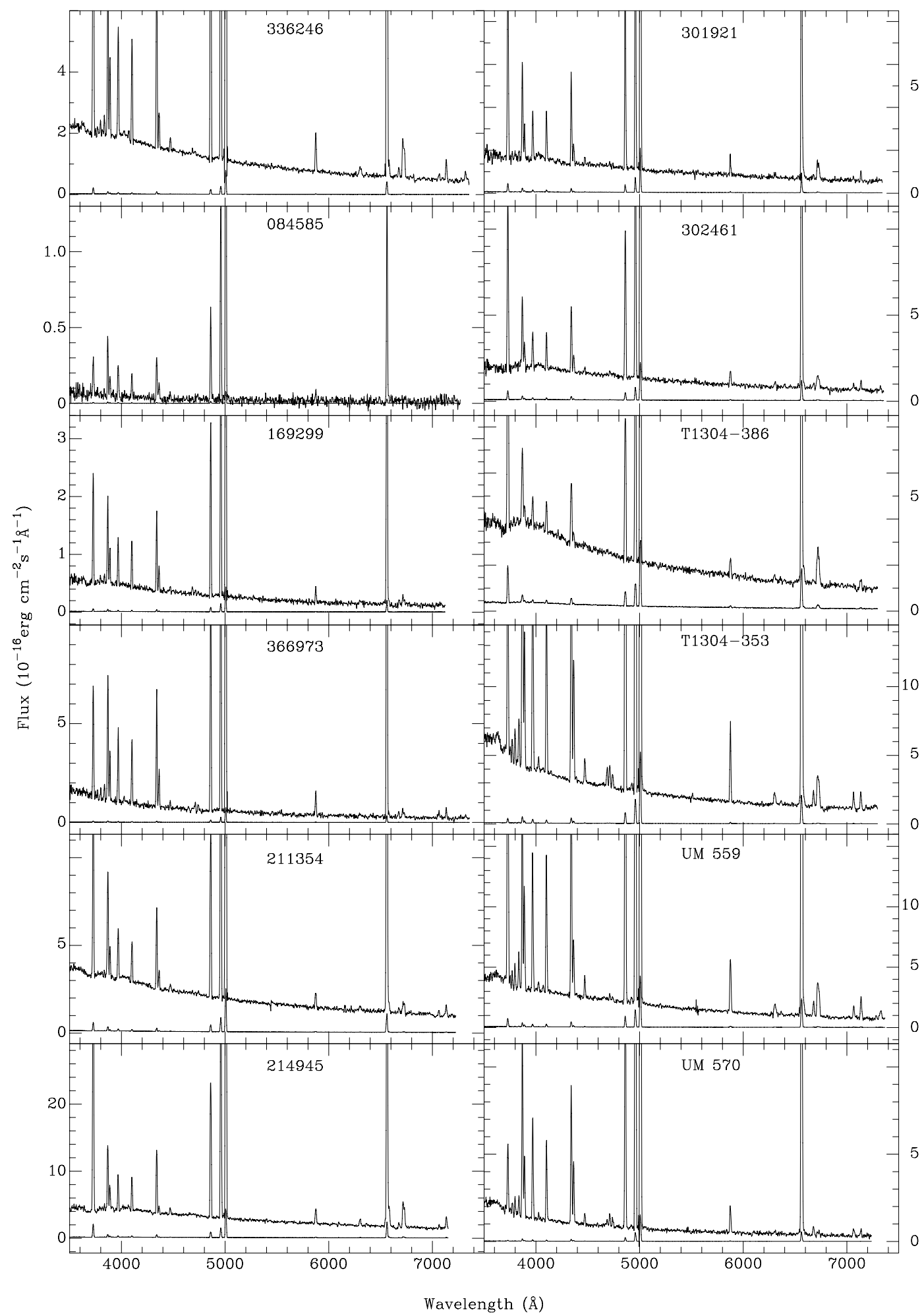

Fig. 2. Spectra of the brightest H II region in each sample galaxy (labeled a in Fig. 1), reduced to zero redshift. In each panel we include the observed spectrum downscaled by different factors for a better visualization of strong emission lines.

signal/noise ratio of the available spectrum. For this galaxy we extracted in addition 1D spectra of three regions, marked in Fig. 1 as b, c and b+c. Note that the weak neon line could not be measured in region $\mathrm{c}$.

Next, we compare our results for the Tololo and UM objects with data from the literature. Using our technique for abundance determination and data for line intensities and equivalent widths from Kehrig et al. (2004), Terlevich et al. (1991) and
Telles \& Terlevich (1995) we recalculated the ionic and element abundances for the Tololo and UM subsample. For some objects, the data from previous studies is not complete. For instance, the [O II] $\lambda 3727$ intensity is not available for UM 570 (Kehrig et al. 2004; Telles \& Terlevich 1995) while the $\mathrm{H} \alpha$ line intensity is not available for UM 570 and Tol 2146-391 (Terlevich et al. 1991). In those cases, we have complemented the literature data by our own, after scaling the measured [O II] $\lambda 3727$ or $\mathrm{H} \alpha$ line 


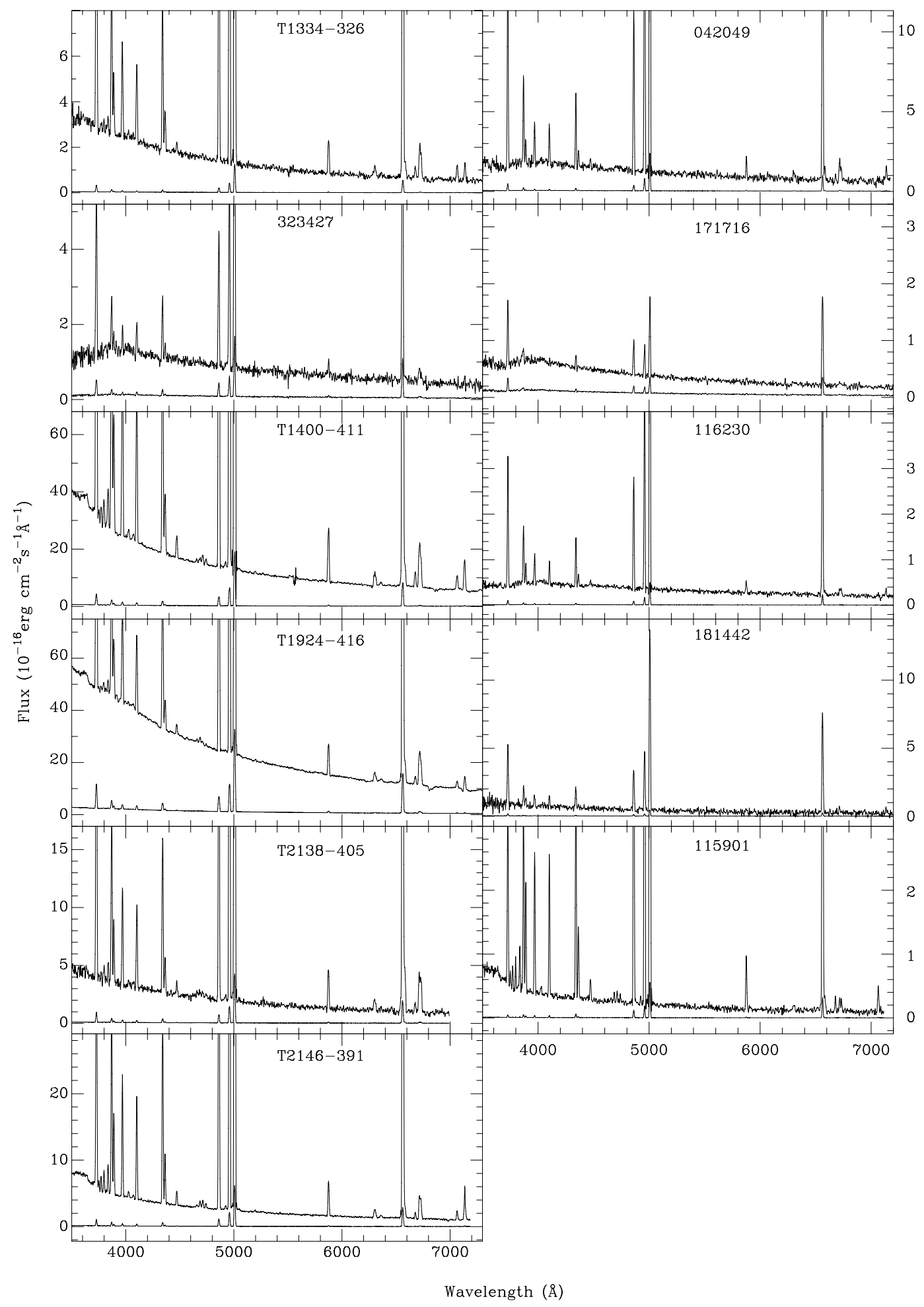

Fig. 2. continued.

intensities relative to $\mathrm{H} \beta$. In Fig. 4 we compare the oxygen (a) and neon (b) abundances for Tololo and UM galaxies with data by Terlevich et al. (1991) (filled small circles), Masegosa et al. (1994) (open circles) and Kehrig et al. (2004) and Telles \& Terlevich (1995) (open triangles). In addition, we compare our oxygen abundance determination for Tol 1304-353 and Tol 1304-386 with that of Kobulnicky \& Skillman (1996) (filled squares), Pagel et al. (1986) (filled triangles) and Campbell (1992) and Marconi et al. (1994) (asterisks), for Tol 1334-326 with Campbell (1992) (asterisk), and for Tol 1400-411 with
Hidalgo-Gámez et al. (2003) (filled star) and Richer \& McCall (1995) (open square). A significant dispersion of the filled small circles about the equality line in Fig. 4 indicates that there exist some discrepancies between our abundances and those calculated from the data of Terlevich et al. (1991). The largest differences ( $\sim .3 \mathrm{dex})$ are seen for Tol 2138-405 and UM 570. Otherwise our determinations are, within formal errors, in agreement with published values.

More specifically, we confirm the low metallicity $(12+$ $\log (\mathrm{O} / \mathrm{H}) \sim 7.7)$ of UM 559 and UM 570 discussed by 
Table 2. Spectroscopic properties of H II regions selected along the slit.

\begin{tabular}{|c|c|c|c|c|c|c|}
\hline H II Region & $t_{\mathrm{e}}\left(\mathrm{O}^{++}\right)^{a}$ & $12+\log (\mathrm{O} / \mathrm{H})$ & $E W(\mathrm{H} \alpha)(\AA)$ & $F(\mathrm{H} \beta)^{b}$ & $C(\mathrm{H} \beta) \operatorname{dex}$ & $100 \times F([\mathrm{O} \mathrm{III}] 4363) / F(\mathrm{H} \beta)$ \\
\hline $336246 a$ & $1.53 \pm 0.02$ & $7.81 \pm 0.01$ & $754 \pm 2$ & $138.6 \pm 0.5$ & $0.060 \pm 0.019$ & $9.9 \pm 0.3$ \\
\hline 084585a & $1.90 \pm 0.08$ & $7.66 \pm 0.04$ & $1531 \pm 18$ & $7.2 \pm 0.1$ & $0.000 \pm 0.034$ & $20.4 \pm 1.5$ \\
\hline $084585 b$ & $1.75 \pm 0.13$ & $7.73 \pm 0.07$ & $893 \pm 22$ & $6.1 \pm 0.2$ & $0.505 \pm 0.056$ & $13.4 \pm 1.9$ \\
\hline $084585 c^{c}$ & $1.62 \pm 0.03$ & $7.72 \pm 0.03$ & $229 \pm 10$ & $2.3 \pm 0.2$ & $0.000 \pm 0.102$ & \\
\hline $169299 a$ & $1.75 \pm 0.04$ & $7.68 \pm 0.02$ & $737 \pm 3$ & $35.8 \pm 0.3$ & $0.000 \pm 0.021$ & $14.5 \pm 0.6$ \\
\hline $169299 \mathrm{~b}^{c}$ & $1.54 \pm 0.10$ & $7.70 \pm 0.09$ & $276 \pm 15$ & $0.5 \pm 0.1$ & $0.000 \pm 0.401$ & \\
\hline $366973 a$ & $1.66 \pm 0.04$ & $7.78 \pm 0.02$ & $1326 \pm 5$ & $92.0 \pm 0.8$ & $0.165 \pm 0.022$ & $14.4 \pm 0.6$ \\
\hline $211354 a$ & $1.41 \pm 0.02$ & $7.95 \pm 0.02$ & $310 \pm 1$ & $94.1 \pm 0.5$ & $0.145 \pm 0.020$ & $10.0 \pm 0.4$ \\
\hline $214945 a$ & $1.11 \pm 0.03$ & $8.23 \pm 0.03$ & $474 \pm 1$ & $137.3 \pm 0.7$ & $0.310 \pm 0.020$ & $4.3 \pm 0.3$ \\
\hline $301921 a$ & $1.69 \pm 0.06$ & $7.76 \pm 0.03$ & $410 \pm 2$ & $53.7 \pm 0.4$ & $0.265 \pm 0.021$ & $13.1 \pm 0.8$ \\
\hline $302461 \mathrm{a}$ & $1.55 \pm 0.04$ & $7.79 \pm 0.02$ & $438 \pm 1$ & $66.7 \pm 0.5$ & $0.250 \pm 0.021$ & $10.1 \pm 0.5$ \\
\hline T1304-386a & $1.46 \pm 0.08$ & $7.89 \pm 0.05$ & $250 \pm 1$ & $55.5 \pm 0.5$ & $0.265 \pm 0.022$ & $7.9 \pm 0.9$ \\
\hline T1304-386b & $1.46 \pm 0.05$ & $7.88 \pm 0.03$ & $639 \pm 3$ & $36.6 \pm 0.3$ & $0.360 \pm 0.022$ & $8.1 \pm 0.6$ \\
\hline T1304-353a & $1.79 \pm 0.02$ & $7.73 \pm 0.01$ & $1424 \pm 2$ & $395.6 \pm 0.9$ & $0.240 \pm 0.019$ & $17.3 \pm 0.3$ \\
\hline UM 559a & $1.58 \pm 0.02$ & $7.72 \pm 0.01$ & $1702 \pm 2$ & $357.2 \pm 0.9$ & $0.240 \pm 0.019$ & $9.6 \pm 0.2$ \\
\hline UM 570a & $1.83 \pm 0.02$ & $7.71 \pm 0.01$ & $1543 \pm 4$ & $172.7 \pm 0.6$ & $0.115 \pm 0.019$ & $20.2 \pm 0.4$ \\
\hline Т1334-326a & $1.49 \pm 0.12$ & $7.92 \pm 0.08$ & $798 \pm 6$ & $105.5 \pm 2.1$ & $0.265 \pm 0.033$ & $11.2 \pm 0.2$ \\
\hline T1334-326b & $1.52 \pm 0.03$ & $7.92 \pm 0.02$ & $1394 \pm 4$ & $83.7 \pm 0.5$ & $0.325 \pm 0.020$ & $11.6 \pm 0.4$ \\
\hline T1334-326c & $1.56 \pm 0.03$ & $7.83 \pm 0.02$ & $1227 \pm 4$ & $73.5 \pm 0.5$ & $0.085 \pm 0.021$ & $11.1 \pm 0.5$ \\
\hline $323427 a$ & $1.47 \pm 0.11$ & $7.76 \pm 0.07$ & $277 \pm 2$ & $31.6 \pm 0.4$ & $0.180 \pm 0.027$ & $7.3 \pm 1.2$ \\
\hline T1400-411a & $1.45 \pm 0.01$ & $7.93 \pm 0.01$ & $1138 \pm 1$ & $1705.0 \pm 2.2$ & $0.230 \pm 0.018$ & $10.2 \pm 0.2$ \\
\hline T1400-411b & $1.41 \pm 0.01$ & $7.95 \pm 0.01$ & $1080 \pm 1$ & $779.7 \pm 1.6$ & $0.205 \pm 0.019$ & $8.7 \pm 0.2$ \\
\hline T1400-411c & $1.10 \pm 0.13$ & $8.09 \pm 0.12$ & $1094 \pm 7$ & $33.1 \pm 0.6$ & $0.070 \pm 0.030$ & $3.8 \pm 0.9$ \\
\hline T1924-416a & $1.42 \pm 0.02$ & $7.94 \pm 0.02$ & $419 \pm 1$ & $973.1 \pm 3.3$ & $0.260 \pm 0.019$ & $8.7 \pm 0.3$ \\
\hline T1924-416b & $1.39 \pm 0.01$ & $7.99 \pm 0.01$ & $655 \pm 1$ & $3273.0 \pm 4.0$ & $0.290 \pm 0.018$ & $8.7 \pm 0.2$ \\
\hline T2138-405a & $1.38 \pm 0.02$ & $7.98 \pm 0.02$ & $1192 \pm 2$ & $269.5 \pm 1.2$ & $0.185 \pm 0.019$ & $8.9 \pm 0.3$ \\
\hline T2146-391a & $1.61 \pm 0.02$ & $7.80 \pm 0.01$ & $1547 \pm 2$ & $460.1 \pm 0.9$ & $0.195 \pm 0.019$ & $12.4 \pm 0.2$ \\
\hline 042049a & $1.28 \pm 0.05$ & $8.08 \pm 0.04$ & $507 \pm 3$ & $82.5 \pm 1.0$ & $0.165 \pm 0.025$ & $8.4 \pm 0.8$ \\
\hline $171716 a$ & $1.69 \pm 0.57$ & $7.54 \pm 0.26$ & $108 \pm 2$ & $6.6 \pm 0.2$ & $0.080 \pm 0.045$ & $5.8 \pm 3.5$ \\
\hline $171716 b$ & $1.80 \pm 0.01$ & $7.47 \pm 0.05$ & $349 \pm 3$ & $8.1 \pm 0.1$ & $0.095 \pm 0.030$ & $8.1 \pm 0.8$ \\
\hline $171716 \mathrm{c}$ & $2.00 \pm 0.57$ & $7.38 \pm 0.20$ & $216 \pm 2$ & $4.0 \pm 0.2$ & $0.175 \pm 0.182$ & $18.1 \pm 7.5$ \\
\hline $171716 b+c$ & $1.78 \pm 0.13$ & $7.45 \pm 0.06$ & $280 \pm 3$ & $16.0 \pm 0.3$ & $0.060 \pm 0.030$ & $7.2 \pm 0.9$ \\
\hline $116230 \mathrm{a}$ & $1.47 \pm 0.06$ & $7.89 \pm 0.04$ & $354 \pm 2$ & $23.1 \pm 0.2$ & $0.080 \pm 0.024$ & $10.6 \pm 0.9$ \\
\hline $181442 a$ & $1.32 \pm 0.02$ & $7.97 \pm 0.12$ & $381 \pm 6$ & $24.7 \pm 0.7$ & $0.165 \pm 0.046$ & $6.4 \pm 2.0$ \\
\hline $115901 \mathrm{a}$ & $1.80 \pm 0.03$ & $7.57 \pm 0.02$ & $2255 \pm 6$ & $70.2 \pm 0.3$ & $0.125 \pm 0.020$ & $12.7 \pm 0.3$ \\
\hline $115901 b$ & $\ldots$ & $\ldots$ & $2250 \pm 950$ & $0.8 \pm 0.4$ & $\ldots$ & $\ldots$ \\
\hline
\end{tabular}

${ }^{a} t_{\mathrm{e}}=10^{-4} T_{\mathrm{e}}$.

${ }^{b}$ Observed flux in units of $10^{-16} \mathrm{erg} \mathrm{s}^{-1} \mathrm{~cm}^{-2}$.

${ }^{c}$ Electron temperature and oxygen abundance obtained with the empirical calibration by Pilyugin (2000).

Salzer et al. (1989a,b) and derived by Kehrig et al. (2004). This is also the case for the oxygen abundance $12+\log (\mathrm{O} / \mathrm{H}) \lessgtr 7.8$ for Tol 2146-391 inferred by Kehrig et al. (2004). With regard to Tol 1304-353 we obtain a relatively low oxygen abundance, $12+\log (\mathrm{O} / \mathrm{H}) \sim 7.7$, in accordance with determinations by Masegosa et al. (1994), Kobulnicky \& Skillman (1996), Pagel et al. (1986) and Campbell (1992).

In summary, our 2dFGRS galaxy sample contains two very metal-deficient systems with an oxygen abundance $12+$ $\log (\mathrm{O} / \mathrm{H}) \leq 7.6$ and another 7 galaxies with oxygen abundance $12+\log (\mathrm{O} / \mathrm{H}) \lesssim 7.8$ (cf. Tables 2 and 5 ). We confirm previous oxygen abundance determinations for Tol 1304-353, Tol 2146-391, UM 559 and UM 570 to be $12+\log (\mathrm{O} / \mathrm{H}) \leq 7.8$.

\subsection{New low-metallicity galaxies from the 2dFGRS}

The present spectroscopic study of the $2 \mathrm{dF}$ Galaxy Redshift Survey led to the discovery of two new extremely metal-deficient emission-line galaxies, $2 \mathrm{dF} 171716$ and $2 \mathrm{dF} 115901$, with an oxygen abundance of $12+\log (\mathrm{O} / \mathrm{H}) \sim 7.5$ and $\sim 7.6$, respectively. Thus, the gap between the three most metal-deficient BCDs known, SBS 0335-052 W\&E $(12+\log (\mathrm{O} / \mathrm{H})=7.12$ and 7.3, Izotov et al. 2005, 1997b), and IZw $18(12+$ $\log (\mathrm{O} / \mathrm{H})=7.2$, Searle \& Sargent 1972; Izotov et al. 1999, and references therein) and the main population of star-forming dwarf galaxies with a mean oxygen abundance $12+\log (\mathrm{O} / \mathrm{H})>$ 7.6 is gradually filling.

The irregular star-forming galaxy $2 \mathrm{dF} 171716$ (Fig. 1t) contains two bright $\mathrm{H}$ II regions located at the southwestern part of its blue, extended high-surface brightness component. In Fig. 5 we include the spectra of four emission-line regions along the slit labeled in Fig. 1t a, b, c and b+c. The electron temperature, ionic and heavy element abundances of the brightest H II region a (labeled 171716a in Fig. 3) are subject to large uncertainties (Table 2). In the second brightest $\mathrm{H}$ II region $\mathrm{b}$, however, where the signal-to-noise is higher we were able to obtain a more reliable oxygen abundance determination. The value $12+\log (\mathrm{O} / \mathrm{H})=7.38 \pm 0.20$ inferred for the extended and by $\sim 0.8$ mag fainter region $\mathrm{c}$ should be considered with caution, as this could be affected by the low S/N [O III] $\lambda 43363 / \mathrm{H} \beta$ intensity ratio. Relatively larger line fluxes and, correspondingly, smaller uncertainties in the heavy element abundances (Table 2) are obtained, however, when a $1 \mathrm{D}$-spectrum is extracted within a larger $1^{\prime \prime} \times 2$ '. 77 aperture, which includes both regions $b$ and $c$ 


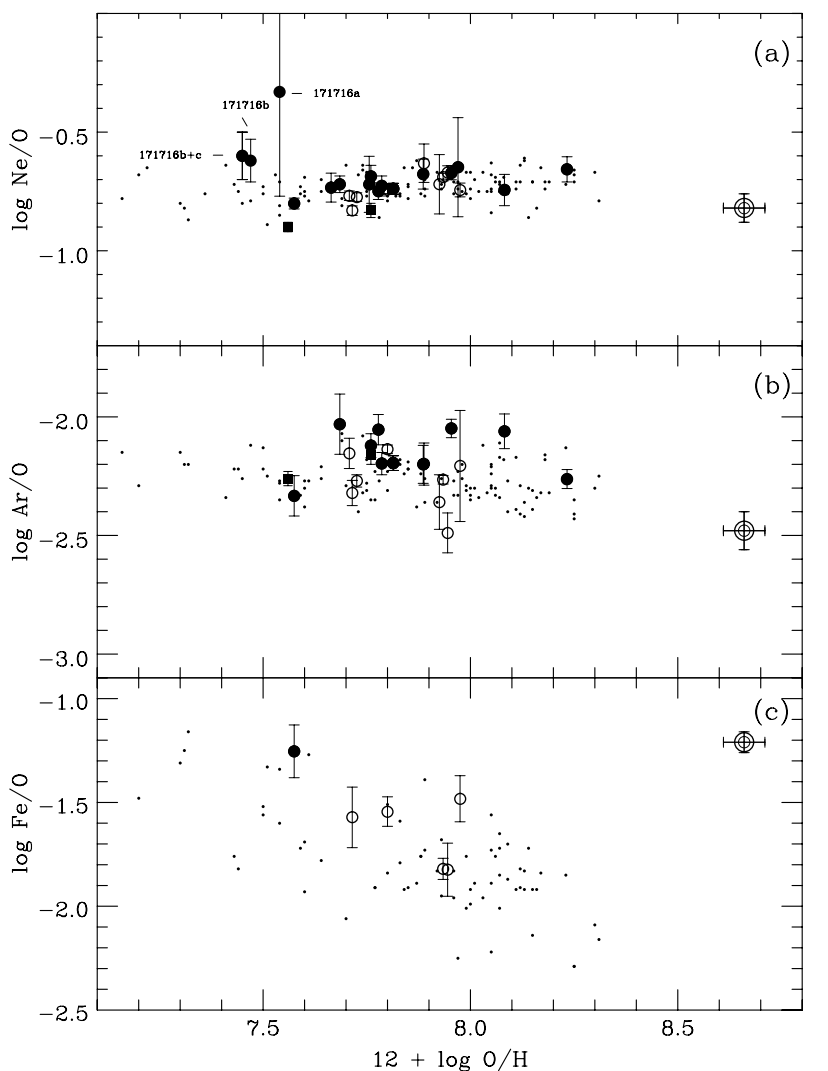

Fig. 3. $\log \mathrm{Ne} / \mathrm{O}$ a), $\log \mathrm{Ar} / \mathrm{O}$ b) and $\log \mathrm{Fe} / \mathrm{O}$ c) vs. oxygen abundance $12+\log \mathrm{O} / \mathrm{H}$ for two samples of emission-line galaxies. Large filled circles show $2 \mathrm{dF}$ galaxies, open circles Tololo and UM galaxies from our sample, and dots galaxies from the comparison sample. Data for Tol 1214-277 (Izotov et al. 2004a) and Pox 186 (Guseva et al. 2004) are shown by filled squares. Three H II regions in the galaxy $2 \mathrm{dF} 171716$ are labeled. Solar ratios from Asplund et al. (2005) are indicated by double large open circles.

(labeled b+c in Fig. 1). From the analysis of all 1D-spectra in Fig. 5, we can constrain the mean oxygen abundance of $2 \mathrm{dF} 171716$ to be $12+\log (\mathrm{O} / \mathrm{H}) \sim 7.5$.

The BCD $2 \mathrm{dF} 115901$ is the second most metal-poor galaxy in our 2dFGRS sample. Its bright $\left(M_{V}=-13.9 \mathrm{mag}\right)$, compact $\mathrm{H}$ II region a (cf. Fig. $1 \mathrm{w}$ ) with an oxygen abundance $12+\log (\mathrm{O} / \mathrm{H})=7.57 \pm 0.02$ is the locus of copious ionized gas emission. The very large equivalent width of the $[\mathrm{O}$ III] $\lambda 5007$ emission line in this region, $E W([\mathrm{O}$ III] $\lambda 5007)=$ $2036 \AA$ (Table 4), suggests that more than $50 \%$ of the $V$-band emission originates from this line. The extreme importance of ionized gas emission to the luminosity budget of region a is also reflected on its measured $E W(\mathrm{H} \alpha)$, exceeding $2200 \AA$. In the adjacent region $\mathrm{b}$, the $\mathrm{H} \beta$ flux is $\sim 90$ times smaller than in region a and the [O III] $\lambda 4363$ emission line could not be detected, while the $E W(\mathrm{H} \alpha)$ remains very large (Table 2). In the absence of direct electron temperature diagnostics, the metallicity of region $b$ cannot be constrained with sufficient precision.

\section{Photometric properties}

The galaxy sample investigated here comprises mainly systems of the $\mathrm{nE}$ and iI morphological BCD types in the Loose \& Thuan (1986) classification scheme. With the exception of Tol 2138-405 $\left(M_{V}=-19.8\right)$ and Tol 1924-416 $\left(M_{V} \approx-19.3\right)$
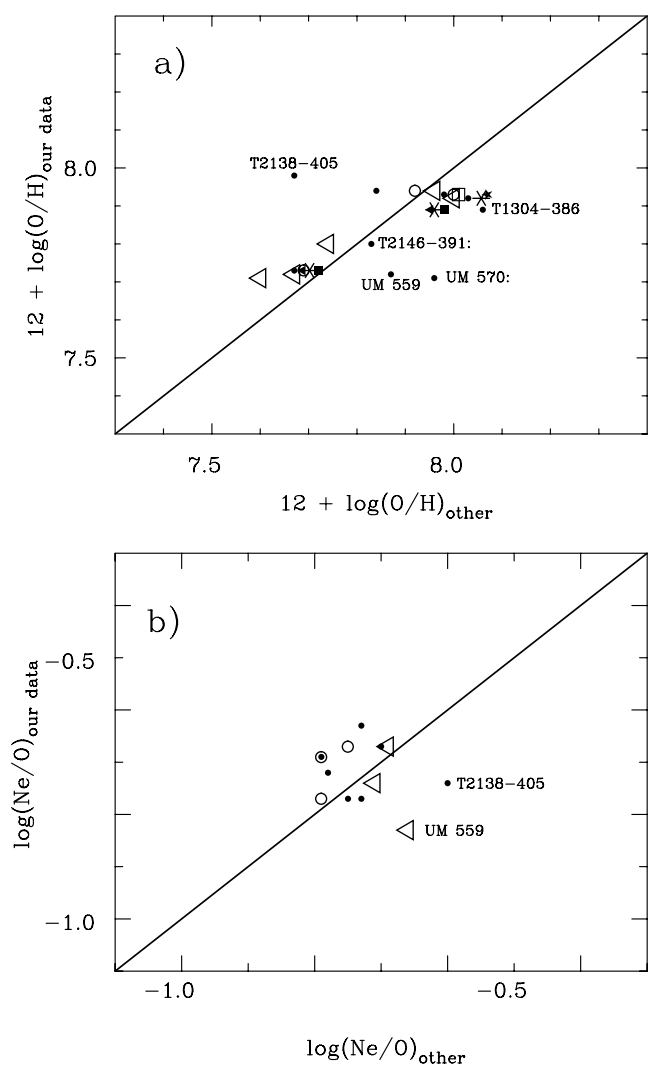

Fig. 4. a) Comparison of our derived oxygen abundances for Tololo and UM galaxies with those of Terlevich et al. (1991) (filled circles), Masegosa et al. (1994) (open circles), Kehrig et al. (2004) and Telles \& Terlevich (1995) (open triangles), Kobulnicky \& Skillman (1996) (filled squares), Pagel et al. (1986) (filled triangles), Campbell (1992) (asterisks), Hidalgo-Gámez et al. (2003) (filled star), Richer \& McCall (1995) (open square). b) The same as in a) but for neon abundances. In both panels, equality between the literature data and ours is indicated by the diagonal line. Galaxies with the largest discrepancies and uncertainties are labeled.

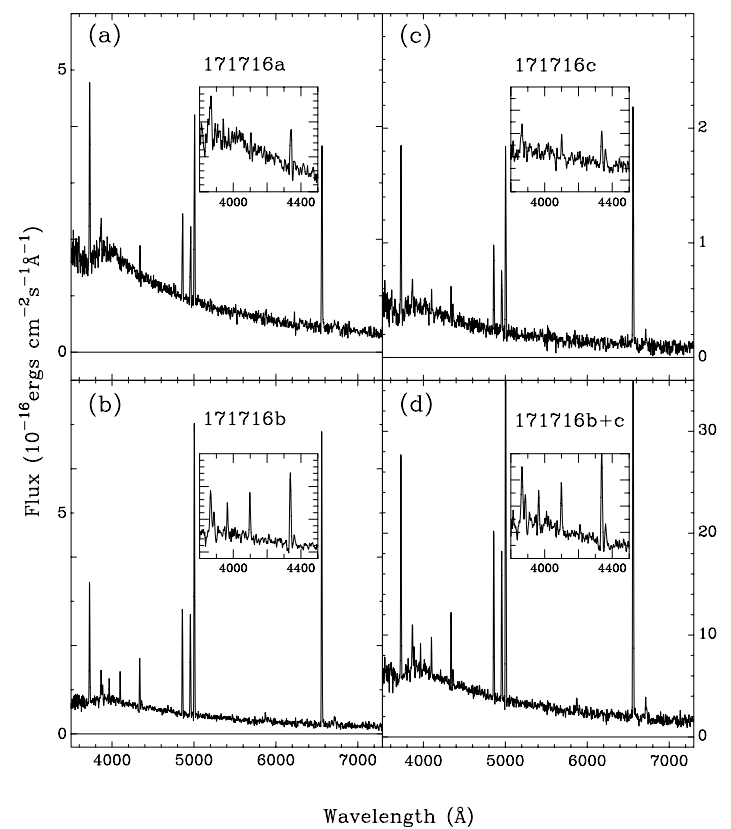

Fig. 5. Extinction-corrected restframe spectra of H II regions in the metal-deficient galaxy 2dF 171716 (cf. Fig. 1t). The insets display enlarged parts of spectra with weak lines, including the [O III] $] 4363 \AA$ emission line. 

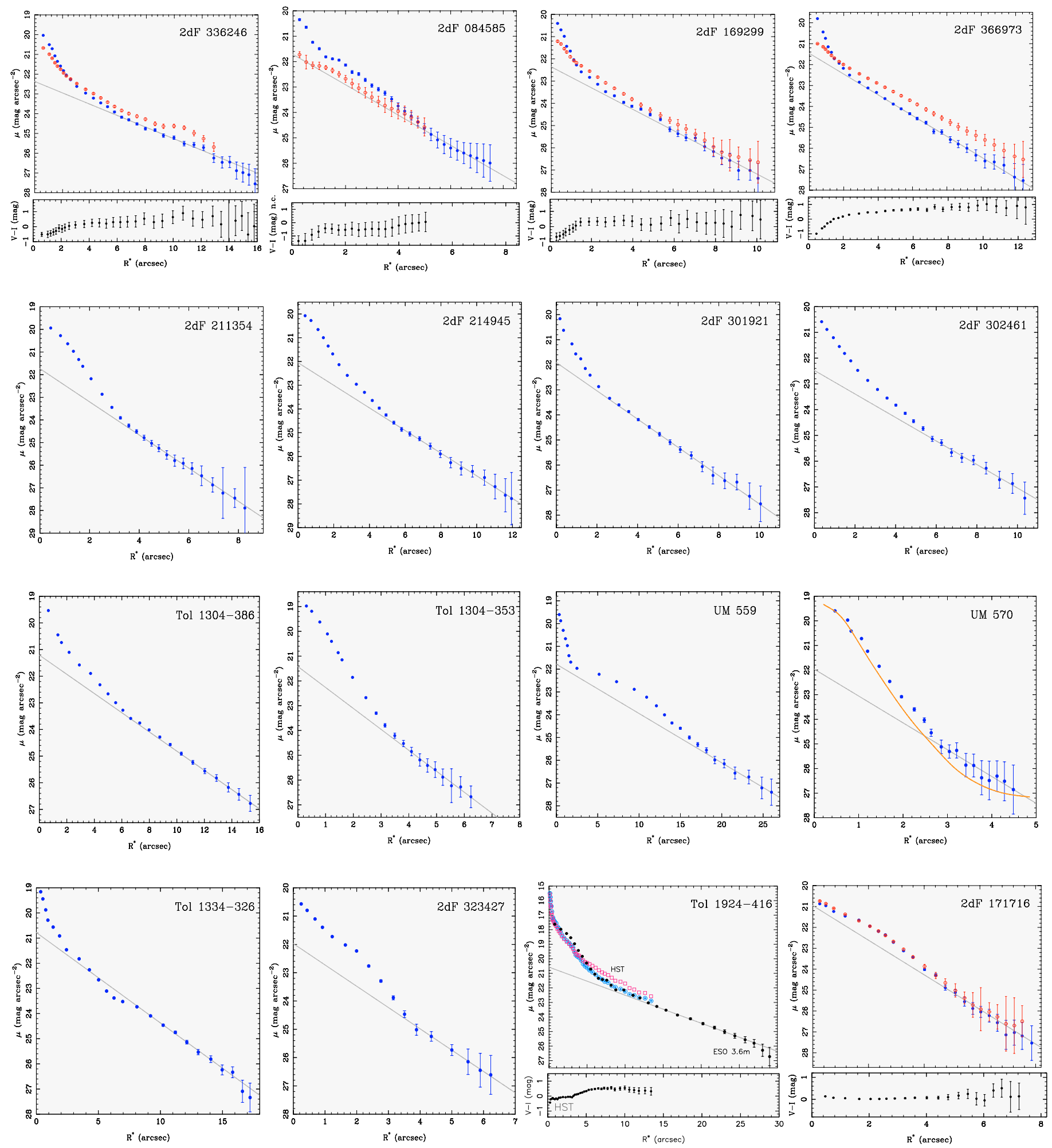

Fig. 6. $V$ band surface brightness profiles (SBPs; filled circles) for all sample galaxies, except for Tol 1400-411 and 2dF 042049. Solid lines show exponential fits to the LSB component (Table 3). Whenever $I$ band data are available (open symbols) we also include radial $V-I$ colour profiles beneath each SBP. For Tol 1924-416, for which only $V$ ground-based data are available, we include SBPs derived from archival HST WFPC2 $V$ and $I$ images. The $I$ SBPs of $2 \mathrm{dF} 116230$ and $2 \mathrm{dF} 181442$ for which no calibration is available, have been shifted vertically such as to match the central intensity in $V$. The uncalibrated $V-I$ profiles of these systems serve only to illustrate colour gradients.

all targets qualify by their absolute magnitude $\left(M_{V}>-18 \mathrm{mag}\right)$ as genuine dwarf galaxies.

This becomes clearer if instead of the total magnitude, one considers only the absolute magnitude $M_{\mathrm{LSB}}$ of the LSB component (Col. 3 in Table 3 ), as derived by exponential fitting to the outer part of each SBP (cf. Fig. 6). Excluding Tol 2138-405, we obtain in that case a mean $M_{\mathrm{LSB}}=-15.5 \pm 1.4$ (with a standard deviation about the mean of $\sigma=0.3$ ), with Tol 1942-416 $\left(M_{\mathrm{LSB}} \approx-18 \mathrm{mag}\right)$ being the intrinsically brightest member in our sample. 

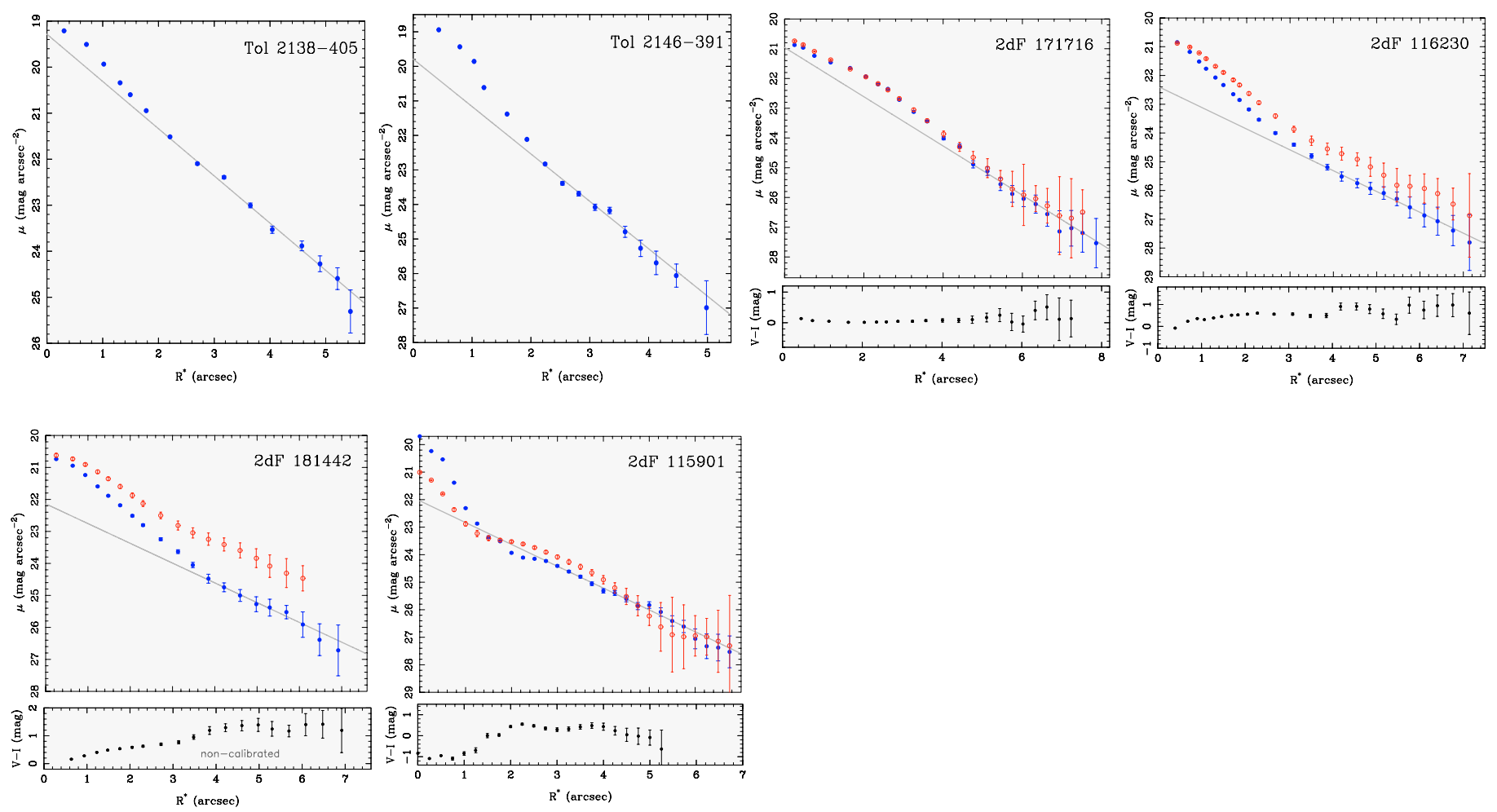

Fig. 6. continued.

In most of our sample galaxies, the SBP of the outer parts of the LSB host is well approximated by an exponential law, as is generally true for dwarf galaxies. The exponential scale length $\alpha$ of the LSB component ranges between $0.2 \mathrm{kpc}$ (2dF 366973) and $1.7 \mathrm{kpc}(2 \mathrm{dF} 169299)$ with a mean value $0.65 \pm 0.4(\sigma=$ $0.09) \mathrm{kpc}$. Thus, our sample contains no ultracompact systems with $\alpha \lesssim 120 \mathrm{pc}$, as for example I Zw 18 (Papaderos et al. 2002) and Pox 186 ( $\alpha=120$ pc, Guseva et al. 2004) but rather displays structural properties typical for BCDs. This is illustrated in Fig. 7 where the present galaxy sample is compared with other dwarf galaxies, such as dwarf irregulars (dIs) and dwarf ellipticals (dEs), in the $M_{\mathrm{LSB}}$ vs. $\log (\alpha)$ plane (see Papaderos et al. 2002, and references therein for details). In doing so, we have assumed throughout an average $B-V$ colour of 0.5 mag for the systems studied here. An overlap between typical BCDs and our sample galaxies is also apparent from the upper panel of the same figure where $M_{\mathrm{LSB}}$ is plotted against the central surface brightness $\mu_{\mathrm{E}, 0}$ of the LSB host galaxy. Similarly, the mean effective radius of $r_{\mathrm{eff}}(\mathrm{kpc})=0.61 \pm 0.31(\sigma=0.09)$ of the present sample matches closely typical values for BCDs, as derived from optical and near infrared surface photometry (e.g., Cairós et al. 2001a; Guseva et al. 2001, 2003; Doublier et al. 2002; Noeske et al. 2003, 2005).

The BCD nature of the emission-line galaxies discussed here is also reflected on the measured ratio of the effective radius $r_{\text {eff }}$ to the scale length $\alpha$ of the LSB component. For a negligible starburst component on top of a purely exponential LSB host, one would expect the observed effective radius to be approximately $1.7 \alpha$. Conversely, a measured ratio $r_{\text {eff }} /(1.7 \alpha)<1$ is indicative of a central luminosity excess on top of the exponential host galaxy, which for the objects under study can be plausibly attributed to the starburst emission. As evident from Fig. 8, the latter ratio is well below unity for all sample galaxies, irrespective of $M_{\mathrm{LSB}}$. The horizontal dashed gray lines in the same figure indicate the expected ratio $r_{\mathrm{eff}} /(1.7 \alpha)$ when a centrally superposed star-forming source approximated here with an exponential component with a scale length $\alpha / 4$, is providing respectively $0 \%, 25 \%, 50 \%$ and $75 \%$ of the total BCD light.

The mean ratio $\left\langle r_{\text {eff }} / 1.7 \alpha\right\rangle=0.62 \pm 0.15(\sigma=0.03)$ for our sample clearly indicates a substantial luminosity contribution from the starburst. Indeed, from profile decomposition we infer the luminosity fraction of the host galaxy to be LSB/total = $0.52 \pm 0.17(\sigma=0.03$; Col. 9 in Table 3$)$ in good agreement with both the low $r_{\mathrm{eff}} /(1.7 \alpha)$ ratio and previous determinations of LSB/total in BCDs based on deep ground-based surface photometry (Papaderos et al. 1996b; Salzer \& Norton 1999; Cairós et al. 2001a; Gil de Paz \& Madore 2005).

In summary, various lines of evidence indicate that most of the emission-line galaxies included in the present study show photometric properties typical for BCDs. That is, in order of importance, i) an LSB host galaxy with $M_{\mathrm{LSB}} \gtrsim-17 \mathrm{~B}$ mag, characterized by a central surface brightness $\mu_{B}<23 \mathrm{mag} / \square^{\prime \prime}$ and a smaller exponential scale length than dIs/dEs of equal $M_{\mathrm{LSB}}$, ii) an optical LSB/total luminosity ratio $\lesssim 0.5$ and iii) an effective radius $r_{\text {eff }} \lesssim 0.6 \mathrm{kpc}$. Additionally, our sample galaxies possess in their majority a smooth circular or elliptical LSB host, as is the case for $\sim 90 \%$ of the local BCD population (the class of BCDs referred to as $\mathrm{iE} / \mathrm{nE}$ in the Loose \& Thuan scheme and numerous systems discovered in the UM survey).

Although spatially resolved colours are available for a few sample galaxies only, we consider it likely that the LSB host in most of the objects investigated here is composed of a several Gyr old stellar population, as is arguably the case for the overwhelming majority of nearby BCDs (Loose \& Thuan 1986; Kunth et al. 1988; Papaderos et al. 1996a; Cairós et al. 2001a; Noeske et al. 2003; Gil de Paz \& Madore 2005, and references therein).

Probable exceptions to this trend are the two most metalpoor galaxies in our sample, $2 \mathrm{dF} 171716$ and $2 \mathrm{dF} 115901$ $(12+\log (\mathrm{O} / \mathrm{H})<7.6)$. The $V-I$ profile of the first system 


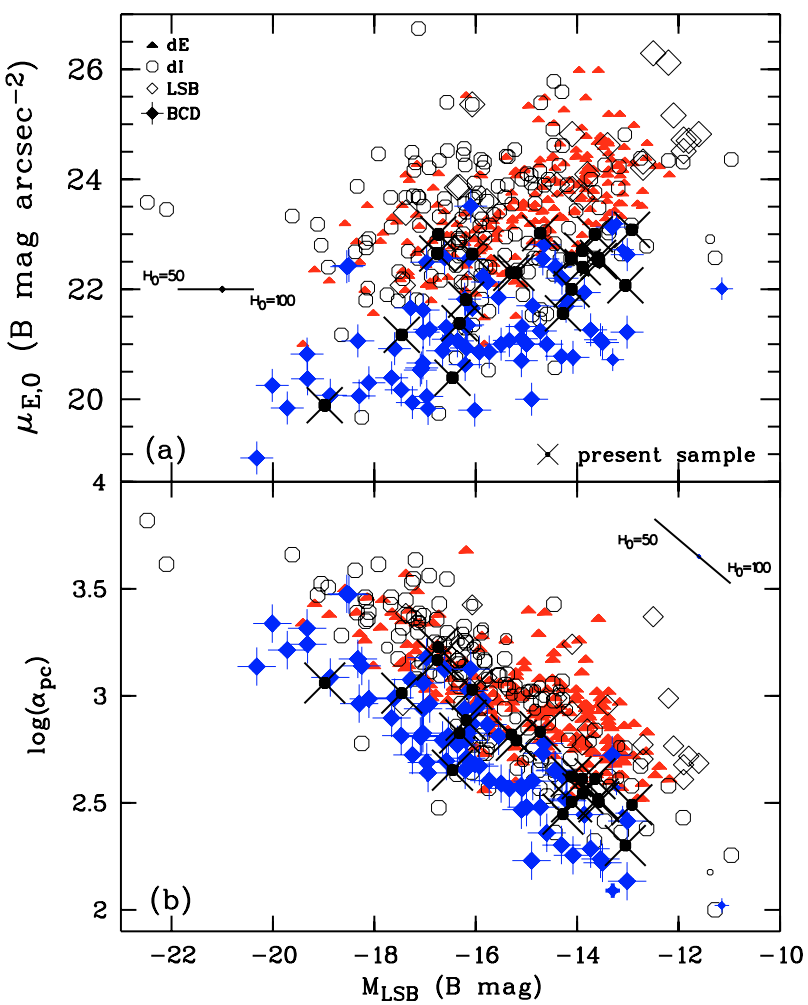

Fig. 7. Comparison of the structural properties of the LSB host of our sample galaxies with those for BCDs and other types dwarf galaxies. Data for iE/nE BCDs are compiled from Cairós et al. (2001a), Drinkwater \& Hardy (1991), Marlowe et al. (1997), Noeske (1999), Noeske et al. (2000) and Papaderos et al. (1996a). Data for other types of dwarf galaxies (dI: dwarf irregulars, dE: dwarf ellipticals, LSB: lowsurface brightness) are taken from Binggeli \& Cameron (1991, 1993), Bothun et al. (1991), Caldwell \& Bothun (1987), Carignan \& Beaulieu (1989), Hopp \& Schulte-Ladbeck (1991), Patterson \& Thuan (1996), Vigroux et al. (1986) and van Zee (2000). Photometric quantities in $V$ have been converted into $B$ assuming a typical $B-V$ colour of 0.5 mag for the LSB component of our sample galaxies. The lines show the shift of the data points caused by a change of the Hubble constant from 75 to 50 and $100 \mathrm{~km} \mathrm{~s}^{-1} \mathrm{Mpc}^{-1}$. a) Central surface brightness $\mu_{\mathrm{E}, 0}$ vs. absolute $B$ magnitude $M_{\mathrm{LSB}}$ of the LSB component. b) Logarithm of the exponential scale length $\alpha$ in pc vs. $M_{\mathrm{LSB}}$.

shows a practically constant colour of $\sim 0.3$ mag, suggesting a uniformly young stellar age out to its Holmberg radius (Fig. 6). Such a colour constancy is very uncommon among BCDs. These systems exhibit almost invariably a strong colour gradient inside their $25 \mathrm{Bmag} / \mathrm{C}^{\prime \prime}$ isophote as a typical signature of the increasing line-of-sight contribution of the underlying old LSB host to the measured intensity with increasing galactocentric radius (see, e.g., Papaderos et al. 1996a, 2002; Gil de Paz \& Madore 2005). The relatively small colour variation within $2 \mathrm{dF} 171716$ with no clear distinction between high-surface brightness starforming regions and the surrounding fainter envelope is also apparent from the colour map in Fig. 9a. This was computed by subtracting the $I$ band image from the $V$ band image after both images were co-registered, smeared to the same angular resolution and calibrated. The colour map is displayed in the range read off the vertical bar to the right of the image $(-0.1 \ldots 0.3 \mathrm{mag})$ and adjusted such that its axis origin coincides with the brightest $\mathrm{H}$ II region a. In order to better compare the broadband morphology with the color distribution we overlay on the $V-I$ map $V$ band contours between 21 and $24.25 \mathrm{mag} \operatorname{arcsec}^{-2}$ in steps of $0.25 \mathrm{mag}$. It is apparent that the colour of $2 \mathrm{dF} 171716$ does

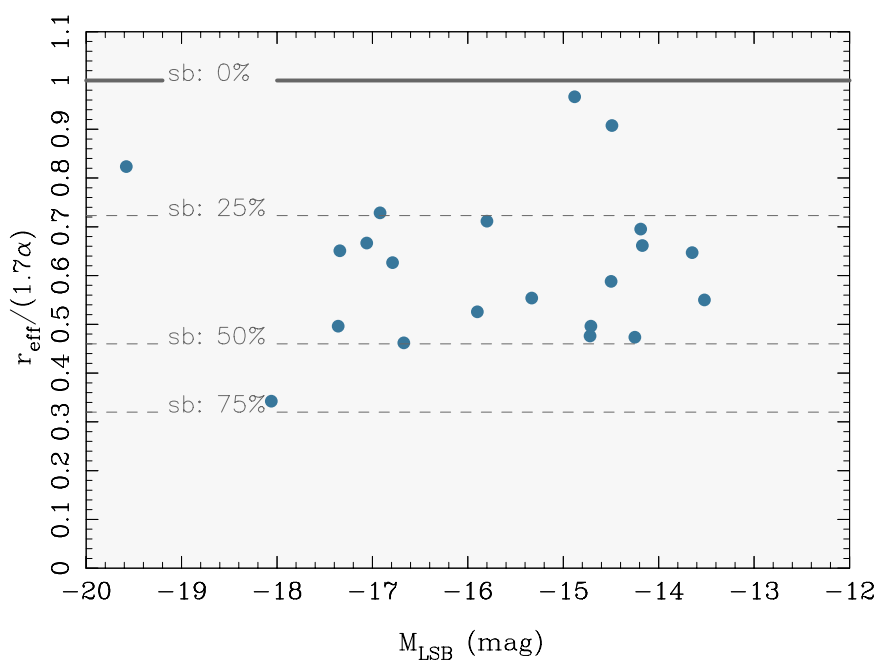

Fig. 8. Absolute $V$ magnitude $M_{\mathrm{LSB}}$ of the host galaxy vs. ratio $r_{\mathrm{eff}} / 1.7 \alpha$, where $r_{\text {eff }}$ denotes the effective radius of our sample galaxies and $\alpha$ is the exponential scale length of the LSB host. A ratio $r_{\text {eff }} / 1.7 \alpha \approx 1$ is expected for a purely exponential intensity profile, that is, in the case when the luminosity contribution of the starburst is negligible. Horizontal lines indicate the expected $r_{\text {eff }} / 1.7 \alpha$ ratio when an exponentially distributed young stellar population with a scale length $\alpha / 4$, providing $25 \%, 50 \%$ and $75 \%$ of the total galaxy's light, is superposed on the LSB component.

not gradually increase towards its LSB periphery, as typically is the case in BCDs, but it shows an irregular pattern with a mean value of $\sim 0.3$ mag. Clearly, deeper spectroscopic and photometric data are needed to decisively check whether or not this emission-line galaxy is embedded within a more extended and faint stellar component with redder colours.

Likewise, the evolutionary status of $2 \mathrm{dF} 115901$ cannot be firmly constrained from the available data. This system displays a strong colour gradient inside $R^{*}=2^{\prime \prime}$ and very blue $V-I$ colours $(\approx-1 \mathrm{mag})$ in its starburst region (see Figs. 6 and $9 \mathrm{~b}$ ). Whereas such a colour index is far too blue to be accounted for by any stellar population, it can readily be explained if optical broadband fluxes are severely contaminated by intense nebular forbidden and Balmer line emission. This is indeed the case here, given that the measured equivalent width of the $[\mathrm{O}$ III $] \lambda 5007$ attains values as large as $\approx 2000 \AA$ (cf. Table 4). While for most star-forming galaxies at $z \approx 0$ corrections of broad band colours for the contribution of ionized gas emission are small ( $\lesssim 0.05 \mathrm{mag}$ in $B-V$, see Salzer et al. 1989a), there are several well-documented cases among BCDs where ample ionized gas emission falsifies colours by more than $0.5 \mathrm{mag}$, not only in the close vicinity of young stellar clusters, but even on a global spatial scale. Most notable examples are the young BCD candidates I Zw 18 (Papaderos et al. 2002) and SBS 0335-052 E (Izotov et al. 1997b; Papaderos et al. 1998).

As apparent from Fig. 6, the radial colour profile of $2 \mathrm{dF} 115901$ levels off for $R^{*} \gtrsim 2 \cdot r_{\text {eff }}$ to a relatively blue mean value of $V-I=0.3 \pm 0.2 \mathrm{mag}$, suggesting the presence of a predominantly young stellar population in its LSB host galaxy. This fact, in connection with a subtle systematic colour variation between the redder northeastern and bluer southwestern part of the LSB component calls for follow-up investigations of the dynamical and evolutionary status of this extremely metal-deficient BCD using deeper data.

A third system whose further investigation is likely of great interest is 2dF 169299 (Fig. 9c). This irregular system displaying 

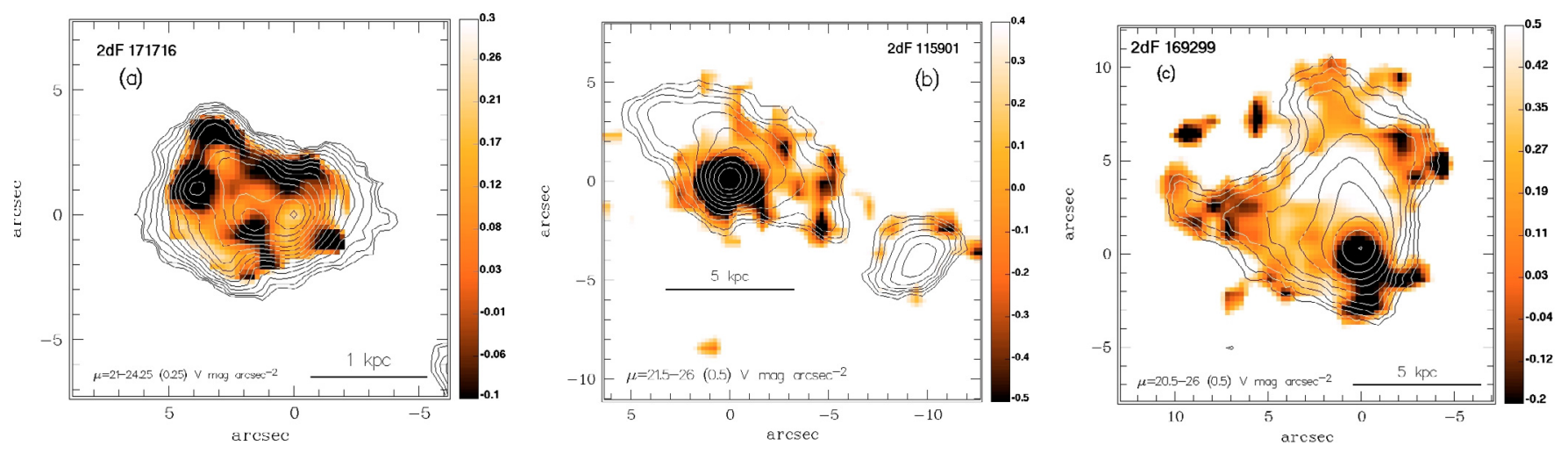

Fig. 9. $V-I$ colour maps of three $2 \mathrm{dFGRS}$ galaxies displayed in the colour range indicated by the respective vertical bars. The surface brightness range of the superposed $V$ band contours and their step size are indicated at the lower-left of each panel. North is to the top and east to the left.

a $\Delta$-like morphology with two fanlike extentions towards NE, bears close resemblance to the BCD II Zw 40 (Sargent \& Searle 1970). These two systems are also fairly similar with regard to their absolute $V$ magnitude ( -17.6 mag for II Zw 40 Cairós et al. 2001a, as compared to -17.7 mag for $2 \mathrm{dF} 169299)$. They differ, however, in the exponential scale length of their LSB component which in the case of $2 \mathrm{dF} 169299$ is twice as large as in II Zw 40 (1.7 kpc as compared to $<0.8 \mathrm{kpc}$; Cairós et al. 2001a; Gil de Paz \& Madore 2005). As evident from the colour map of the BCD the brightest star-forming region a $(V-I \approx-0.3)$ is surrounded by blue $(0 \ldots 0.1 \mathrm{mag})$ patches along the northeastern extentions and at the position of region $\mathrm{b}$. The $V-I$ colour in the LSB component is comparatively blue, $\sim 0.4 \mathrm{mag}$, possibly indicating a relatively young evolutionary state, provided that extended ionized gas emission does not significantly affect colours in the outskirts of this system.

Clearly, the sensitivity and photometric accuracy of the available broadband data are not sufficient for a conclusive study of the evolutionary status of the three aforementioned BCDs. This kind of analysis was outside the scope and technical design of our observations, which have been solely optimized for the search of metal-deficient emission-line galaxies. In view of the present results, however, a closer investigation of the latter systems using deep follow-up imagery and spatially resolved spectroscopy is apparently of great interest.

\section{Summary}

We have extracted from the Two-Degree Field Galaxy Redshift Survey (2dFGRS) 100 K Data Release a sample of 14 emissionline galaxies with a relatively strong [O III] $\lambda 4363$ emission line which appear to be promising candidates for being new lowmetallicity star-forming galaxies. Spectroscopic and photometric studies of this sample, and of 7 Tololo and 2 UM additional galaxies were carried out using observations obtained with the ESO $3.6 \mathrm{~m}$ telescope at La Silla.

For the majority of our sample galaxies, broad-band imaging reveals a low-surface brightness (LSB) host galaxy extending well beyond the star-forming component. As surface photometry in $V$ for all objects and in $I$ for some objects indicates, this LSB host is well approximated by an exponential fitting law and provides approximately half of the total $V$ emission, as is generally the case for blue compact dwarf (BCD) galaxies. Moreover, our photometric analysis indicates that the spectroscopically selected galaxies studied here are indistinguishable from BCDs regarding the structural properties (e.g., central surface brightness and exponential scale length) of their LSB host. These facts establish a posteriori the BCD nature of our sample galaxies.

Based on longslit spectroscopy, we identify seven $2 \mathrm{dF}$ galaxies with an oxygen abundance $12+\log (\mathrm{O} / \mathrm{H}) \lesssim 7.8$, and confirm previous claims that the star-forming systems Tol 1304-353, Tol 2146-391, UM 559 and UM 570 also have $12+\log (\mathrm{O} / \mathrm{H}) \leq 7.8$. Additionally, we find that the distributions of our sample galaxies in the $12+\log (\mathrm{O} / \mathrm{H})$ vs. $\log (\mathrm{Ne} / \mathrm{O})$, $\log (\mathrm{Ar} / \mathrm{O})$ and $\log (\mathrm{Fe} / \mathrm{O})$ diagrams are similar to those of a comparison sample of $\sim 100$ emission-line galaxies from Izotov et al. (2004b).

Most importantly, we report the discovery of two galaxies, $2 \mathrm{dF} 115901$ and $2 \mathrm{dF} 171716$, with an oxygen abundance $12+\log (\mathrm{O} / \mathrm{H}) \sim 7.6$ and $\sim 7.5$, respectively. This allows us to go another step forward in filling the gap between the most metal-deficient star-forming galaxies known SBS 0335$052 \mathrm{~W} \& \mathrm{E}(12+\log (\mathrm{O} / \mathrm{H})=7.1$ and 7.3 , respectively $)$ and I Zw $18(12+\log (\mathrm{O} / \mathrm{H})=7.2)$ and the majority of BCDs with a mean oxygen abundance $12+\log (\mathrm{O} / \mathrm{H})>7.6$.

Acknowledgements. N.G.G. and Y.I.I. have been supported by DFG grants 436 UKR 17/2/04 and 436 UKR 17/25/05. The research described in this paper was made possible in part by Award No. UP1-2551-KV-03 of the US Civilian Research \& Development Foundation for the Independent States of the Former Soviet Union (CRDF). They are grateful for the hospitality of the Göttingen Observatory. T.X.T. and Y.I.I. have been partially supported by NSF grant AST-02-05785. T.X.T. is grateful for a Sesquicentennial Fellowship from the University of Virginia. He thanks the hospitality of the Institut d'Astrophysique in Paris and of the Service d'Astrophysique at Saclay during his sabbatical leave. This research has made use of the NASA/IPAC Extragalactic Database (NED) which is operated by the Jet Propulsion Laboratory, California Institute of Technology, under contract with the National Aeronautics and Space Administration. P.P. and K.G.N. would like to thank Linda Schmidtobreick, José Cortés, Olivier Hainaut, George Hau, Erich Wenderoth and the whole ESO staff at the La Silla Observatory for their support.

\section{References}

Asplund, M., Grevesse, N., \& Sauval, A. J. 2005, in Cosmic Abundances as Records of Stellar Evolution and Nucleosynthesis, ASP Conf. Ser., 336, 25 Bicker, J., Fritze-v. Alvensleben, U., Möller, C. S., \& Fricke, K. J. 2004, A\&A, 413, 37

Binggeli, B., \& Cameron, L. M. 1991, A\&A, 252, 27

Binggeli, B., \& Cameron, L. M. 1993, A\&AS, 98, 297

Bothun, G. D., Impey, C. D., \& Malin, D. F. 1991, ApJ, 376, 404

Cairós, L. M., Vílchez, J. M., González Pérez, J. N., Iglesias-Páramo, J., \& Caon, N. 2001a, ApJS133, 321

Cairós, L. M., Caon, N., Vílchez, J. M., González Pérez, J. N., \& Muñoz-Tuñón, C. 2001b, ApJS, 133, 321

Caldwell, N., \& Bothun, G. D. 1987, AJ, 94, 1126

Campbell, A. 1992, ApJ, 401, 157 
Campbell, A. W., Terlevich, R., \& Melnick, J. 1986, MNRAS, 223, 811

Carignan, C., \& Beaulieu, S. 1989, ApJ, 347, 760

Colless, M., Dalton, G., Maddox, S., et al. 2001, MNRAS, 328, 1039

Doublier, V., Caulet, A., \& Comte, G. 2002, A\&A, 367, 33

Drinkwater, M., \& Hardy, E. 1991, AJ, 101, 94

Fioc, M., \& Rocca-Volmerange, B. 1997, A\&A, 326, 950

Fricke, K. J., Izotov, Y. I., Papaderos, P., Guseva, N. G., \& Thuan, T. X. 2001, AJ, 121, 169

Gil de Paz, A., \& Madore, B. F. 2005, ApJS, 156, 345

Gil de Paz, A., Madore, B. F., \& Pevunova, O. 2003, ApJS, 147, 29

Guseva, N. G., Izotov, Y. I., Papaderos, P., et al. 2001, A\&A, 378, 756

Guseva, N. G., Papaderos, P., Izotov, Y. I., et al. 2003, A\&A, 407, 75

Guseva, N. G., Papaderos, P., Izotov, Y. I., Noeske, K. G., \& Fricke, K. J. 2004, A\&A, 421, 519

Hidalgo-Gámez, A. M., Sánchez-Salcedo, F. J., \& Olofsson, K. 2003, A\&A, 399, 63

Hopp, U., \& Schulte-Ladbeck, R. E. 1991, A\&A, 248, 1

Izotov, Y. I., \& Thuan, T. X. 1998, ApJ, 497, 227

Izotov, Y. I., \& Thuan, T. X. 2004a, ApJ, 602, 200

Izotov, Y. I., \& Thuan, T. X. 2004b, ApJ, 616, 768

Izotov, Y. I., Thuan, T. X., \& Lipovetsky, V. A. 1994, ApJ, 435, 647

Izotov, Y. I., Thuan, T. X., \& Lipovetsky, V. A. 1997a, ApJS, 108, 1

Izotov, Y. I., Lipovetsky, V. A., Chaffee, F. H., et al. 1997b, ApJ, 476, 698

Izotov, Y. I., Chaffee, F. H., Foltz, C. B., et al. 1999, ApJ, 527, 757

Izotov, Y. I., Chaffee, F. H., \& Schaerer, D. 2001, A\&A, 378, L45

Izotov, Y. I., Noeske, K. G., Guseva, N. G., et al. 2004a, A\&A, 415, L27

Izotov, Y. I., Stasińska, G., Guseva, N. G., \& Thuan, T. X. 2004b, A\&A, 415, 87

Izotov, Y. I., Thuan, T. X., \& Guseva, N. G. 2005, ApJ, 632, 210

Izotov, Y. I., Stasińska, G., Meynet, G., Guseva, N. G., \& Thuan, T. X. 2006, A\&A, 448, 955

Kehrig, K., Telles, E., \& Cuisinier, F. 2004, AJ, 128, 1141

Kobulnicky, H. A., \& Skillman, E. D. 1996, ApJ, 471, 211

Kunth, D., Maurogordato, S., \& Vigroux, L. 1988, A\&A, 204, 10

Kraan-Korteweg, R. C. 1986, A\&AS, 66, 255

Landolt, A. U. 1992, AJ, 104, 340

Lauberts, A., \& Valentijn, E. A. 1989, The Surface Photometry Catalogue of the ESO-Uppsala Galaxies, ESO

Loose, H. H., \& Thuan, T. X. 1986, Star Forming Dwarf Galaxies and Related Objects (Éditions Frontières), 73

Maddox, S. J., Sutherland, W. J., Efstathiou, G., \& Loveday, J. 1990, MNRAS, 243,692

Marconi, G., Matteucci., F., \& Tosi, M. 1994, MNRAS, 270, 35
Marlowe, A. T., Meurer, G. R., Heckman, T. M., \& Schommer, R. 1997, ApJS, 112,285

Masegosa, J., Moles, M., \& Campos-Aguilar, A. 1994, ApJ, 420, 576

Noeske, K. G. 1999, Diploma Thesis

Noeske, K. G., Guseva, N. G., Fricke, K. J., et al. 2000, A\&A, 361, 33

Noeske, K. G., Papaderos, P., Cairós, L. M., \& Fricke, K. J. 2003, A\&A, 410, 481

Noeske, K. G., Papaderos, P., Cairós, L. M., \& Fricke, K. J. 2005, A\&A, 429, 115

Pagel, B. E. J., Terlevich, R. J., \& Melnick, J. 1986, MNRAS, 98, 1005

Pagel, B. E. J., Simonson, E. A., Terlevich, R. J., \& Edmunds, M. G. 1992, MNRAS, 255, 325

Papaderos, P., Loose, H.-H., Thuan, T. X., \& Fricke, K. J. 1996a, A\&AS, 120, 207

Papaderos, P., Loose, H.-H., Fricke, K. J., \& Thuan, T. X. 1996b, A\&A, 314, 59 Papaderos, P., Izotov, Y. I., Fricke, K. J., Thuan, T. X., \& Guseva, N. G. 1998, A\&A, 338, 43

Papaderos, P., Izotov, Y. I., Thuan, T. X., et al. 2002, A\&A, 292, 461

Patterson, R. J., \& Thuan, T. X. 1996, ApJS, 107, 103

Peimpert, M., \& Torres-Peimpert, S. 1974, ApJ, 193, 327

Peimpert, M., \& Torres-Peimpert, S. 1976, ApJ, 203, 581

Petrosian, V. 1976, ApJ, 210, L53

Pilyugin, L. S. 2000, A\&A, 362, 325

Pustilnik, S. A., Pramskij, A. G., \& Kniazev, A. Y. 2004, A\&A, 425, 51

Richer, M. G., \& McCall, M. L. 1995, ApJ, 445, 642

Salzer, J. J., MacAlpine, G. M., \& Boroson, T. A. 1989a, ApJS, 70, 447

Salzer, J. J., MacAlpine, G. M., \& Boroson, T. A. 1989b, ApJS, 70, 479

Salzer, J. J., \& Norton, S. A. 1999, in the Low Surface Brightness Universe, ASP Conf. Ser., 170, 253

Sargent, W. L. W., \& Searle, L. 1970, ApJ, 152, L155

Schlegel, D. J., Finkbeiner, D. P., \& Davis, M. 1989, ApJ, 500, 525

Searle, L., \& Sargent, W. L. W. 1972, ApJ, 173, 25

Stasińska, G., \& Izotov, Y. I. 2003, A\& A, 397, 71

Takamiya, M. 1999, ApJS, 122, 109

Telles, E., \& Terlevich, R. 1995, MNRAS, 275, 1

Terlevich, R., Melnick, J., Masegosa, J., Moles, M., \& Copetti, M. V. F. 1991, A\&AS, 91, 285

Thuan, T. X., \& Izotov, Y. I. 2005, ApJS, 161, 240

Thuan, T. X., Izotov, Y. I., \& Lipovetsky, V. A. 1995, ApJ, 445, 108

van Zee, L. 2000, AJ, 119, 2757

van Zee, L., Skillman, E. D., \& Haynes, M. P. 2004, AJ, 128, 121

Vigroux, L., Thuan, T. X., Vader, J. P., \& Lachièze-Rey, M. 1986, AJ, 91, 70 
P. Papaderos et al.: New southern blue compact dwarf galaxies in the $2 \mathrm{dF}$ Galaxy redshift survey, Online Material p 1

\section{Online Material}


P. Papaderos et al.: New southern blue compact dwarf galaxies in the $2 \mathrm{dF}$ Galaxy redshift survey, Online Material p 2

Table 3. Structural properties of the sample galaxies in the $V$ band.

\begin{tabular}{|c|c|c|c|c|c|c|c|c|c|}
\hline $\begin{array}{l}\text { Name } \\
\text { morph. type } \dagger\end{array}$ & $\begin{array}{c}\mu_{\mathrm{E}, 0} \\
\alpha\end{array}$ & $\begin{array}{l}m_{\mathrm{LSB}} \\
M_{\mathrm{LSB}}\end{array}$ & $\begin{array}{l}m \\
M\end{array}$ & $\begin{array}{r}r_{\mathrm{eff}} \\
\mu_{\mathrm{eff}}\end{array}$ & $\begin{array}{c}r_{\text {Petr }} \\
m_{\text {Petr }}\end{array}$ & $\begin{array}{c}\log \left(r_{80} / r_{20}\right) \\
1 / \eta\end{array}$ & $\begin{array}{c}r_{25} / r_{\text {eff }} \\
\text { fit range }\end{array}$ & $\begin{array}{l}\text { LSB/total } \\
(V-I)_{\mathrm{LSB}}\end{array}$ & $\begin{array}{l}D \\
A_{\mathrm{V}}\end{array}$ \\
\hline (1) & (2) & (3) & (4) & (5) & (6) & (7) & (8) & (9) & $(10)$ \\
\hline $2 \mathrm{dF} 336246$ & $22.4 \pm 0.1$ & 17.51 & 16.95 & 0.33 & 0.45 & 0.82 & 3.0 & 0.57 & 22.7 \\
\hline $\mathrm{nE}$ & $0.41 \pm 0.02$ & -14.25 & -14.83 & 21.31 & 17.50 & 0.38 & $6^{\prime \prime}-16^{\prime \prime} 9$ & $0.55 \pm 0.06$ & 0.03 \\
\hline $2 \mathrm{dF} 084585$ & $21.7 \pm 0.8$ & 18.42 & 17.9 & 0.75 & 1.27 & 0.60 & 0.49 & 0.60 & 69.7 \\
\hline iI & $0.62 \pm 0.15$ & -15.80 & -16.31 & 21.63 & 18.15 & 0.49 & 4.. $8-7 . .6$ & - & 0.05 \\
\hline $2 \mathrm{dF} 169299$ & $22.4 \pm 0.4$ & 18.57 & 18.22 & 1.87 & 3.42 & 0.79 & 2.24 & 0.72 & 152.5 \\
\hline iI & $1.69 \pm 0.2$ & -17.34 & -17.70 & 22.23 & 18.54 & 0.46 & $5.6-10.7$ & $0.38 \pm 0.07$ & 0.06 \\
\hline $2 \mathrm{dF} 366973$ & $21.47 \pm 0.14$ & 17.77 & 17.40 & 0.22 & 0.39 & 0.84 & 2.95 & 0.71 & 19.25 \\
\hline $\mathrm{nE}$ & $0.2 \pm 0.01$ & -13.65 & -14.02 & 21.28 & 17.78 & 0.50 & $5^{\prime \prime}-12^{\prime \prime} 6$ & $0.78 \pm 0.03$ & 0.07 \\
\hline $2 \mathrm{dF} 211354$ & $21.7 \pm 0.35$ & 18.9 & 17.7 & 0.59 & 0.93 & 0.54 & 3.44 & 0.34 & 92 \\
\hline $\mathrm{nE}$ & $0.66 \pm 0.06$ & -15.9 & -17.2 & 20.26 & 17.99 & 0.54 & $4^{\prime \prime}-8{ }^{\prime \prime} 3$ & - & 0.12 \\
\hline $2 \mathrm{dF} 214945$ & $22.05 \pm 0.3$ & 18.27 & 17.37 & 1.24 & 1.72 & 0.64 & 3.2 & 0.43 & 133.5 \\
\hline iI & $1.47 \pm 0.13$ & -17.36 & -18.26 & 20.78 & 17.86 & 0.54 & $4^{\prime \prime}-12^{\prime \prime}$ & - & 0.16 \\
\hline $2 \mathrm{dF} 301921$ & $21.91 \pm 0.14$ & 18.49 & 18.18 & 0.36 & 0.47 & 0.73 & 2.5 & 0.73 & 34.1 \\
\hline $\mathrm{nE}$ & $0.32 \pm 0.02$ & -14.17 & -14.48 & 21.87 & 18.74 & 0.55 & $4^{\prime \prime}-10^{\prime \prime}$ & - & 0.07 \\
\hline $2 \mathrm{dF} 302461$ & $22.48 \pm 0.36$ & 18.6 & 17.89 & 0.29 & 0.45 & 0.65 & 2.48 & 0.48 & 26.5 \\
\hline $\mathrm{nE}$ & $0.31 \pm 0.04$ & -13.52 & -14.23 & 21.67 & 18.29 & 0.61 & $5.7-10 .{ }^{\prime} 4$ & - & 0.1 \\
\hline Tol 1304-386 & $21.21 \pm 0.44$ & 16.82 & 16.24 & 0.82 & 1.31 & 0.72 & 3.3 & 0.57 & 52.6 \\
\hline iI & $0.77 \pm 0.09$ & -16.79 & -17.36 & 20.77 & 16.61 & 0.58 & $10.9-15^{\prime \prime} 3$ & - & 0.1 \\
\hline Tol 1304-353 & $21.4 \pm 0.7$ & 18.88 & 17.34 & 0.27 & 0.43 & 0.54 & 4.0 & 0.24 & 52.1 \\
\hline $\mathrm{nE}$ & $0.32 \pm 0.06$ & -14.71 & -16.25 & 19.46 & 17.66 & 0.61 & 3.. 9-6". 3 & - & 0.185 \\
\hline UM 559 & $21.8 \pm 0.8$ & 16.28 & 15.64 & 0.54 & 0.87 & 0.51 & 2.07 & 0.54 & 14.3 \\
\hline $\mathrm{iE} / \mathrm{iI}$ & $0.35 \pm 0.07$ & -14.49 & -15.14 & 22.09 & 23.35 & 0.84 & $19^{\prime \prime}-26^{\prime \prime}$ & - & 0.09 \\
\hline UM 570 & $21.96 \pm 0.55$ & 19.98 & 18.10 & 0.34 & 0.64 & 0.49 & 3.54 & 0.17 & 87.1 \\
\hline $\mathrm{nE}$ & $0.42 \pm 0.08$ & -14.72 & -16.6 & 19.58 & 18.29 & 0.52 & $2 . .8-3^{\prime \prime} .7$ & - & 0.10 \\
\hline Tol 1334-326 & $20.78 \pm 0.14$ & 16.38 & 16.21 & 0.83 & 1.14 & 0.74 & 3.2 & 0.8 & 45.8 \\
\hline $\mathrm{iI}, \mathrm{C}$ & $0.67 \pm 0.03$ & -16.92 & -17.1 & 21.07 & 16.7 & 0.58 & $9^{\prime \prime}-17^{\prime \prime}$ & - & 0.17 \\
\hline $2 \mathrm{dF} 323427$ & $21.97 \pm 0.8$ & 19.18 & 18.34 & 0.39 & 0.62 & 0.52 & 2.3 & 0.44 & 47.2 \\
\hline iI,C & $0.33 \pm 0.06$ & -14.19 & -15.03 & 21.48 & 18.59 & 0.87 & $4^{\prime \prime}-6 . ' 3$ & - & 0.16 \\
\hline Tol 1924-416 & $20.57 \pm 0.15$ & 14.82 & 13.62 & 0.60 & 0.81 & 0.67 & 7.03 & 0.33 & 37.8 \\
\hline $\mathrm{iE}$ & $1.03 \pm 0.04$ & -18.06 & -19.27 & 18.19 & 14.11 & 0.36 & $14^{\prime \prime} 7-28^{\prime \prime}$ & - & 0.29 \\
\hline Tol 2138-405 & $19.29 \pm 34$ & 17.17 & 16.95 & 1.61 & 2.51 & 0.57 & 3.6 & 0.82 & 224 \\
\hline $\mathrm{nE} / \mathrm{iI}$ & $1.15 \pm 0.1$ & -19.58 & -19.80 & 19.84 & 17.34 & 0.94 & 3.. 4-5". 5 & - & 0.07 \\
\hline Tol 2146-391 & $19.79 \pm 0.36$ & 18.30 & 17.38 & 0.51 & 0.75 & 0.52 & 4.2 & 0.42 & 118 \\
\hline $\mathrm{nE}$ & $0.45 \pm 0.04$ & -17.06 & -17.99 & 19.12 & 17.73 & 0.70 & $2{ }^{\prime \prime} 7-5^{\prime \prime}$ & - & 0.1 \\
\hline $2 \mathrm{dF} 171716$ & $20.96 \pm 0.6$ & 18.37 & 17.89 & 0.46 & 0.74 & 0.47 & 2.33 & 0.64 & 44.7 \\
\hline iI & $0.28 \pm 0.04$ & -14.88 & -15.36 & 21.52 & 18.14 & 1.01 & 4." $6-8$ " & $0.2 \pm 0.06$ & 0.08 \\
\hline $2 \mathrm{dF} 116230$ & $22.42 \pm 0.53$ & 19.54 & 18.73 & 0.64 & 0.99 & 0.62 & 2.61 & 0.47 & 94.2 \\
\hline $\mathrm{nE}$ & $0.68 \pm 0.08$ & -15.33 & -16.14 & 21.48 & 19.12 & 0.60 & 3..7-7".'1 & $0.72 \pm 0.07$ & 0.07 \\
\hline $2 \mathrm{dF} 181442$ & $22.1 \pm 0.5$ & 18.9 & 18.2 & 0.41 & 0.63 & 0.57 & 2.6 & 0.51 & 48.2 \\
\hline $\mathrm{nE}$ & $0.41 \pm 0.08$ & -14.5 & -15.2 & 21.4 & 18.6 & 0.78 & 4."4-7" & - & 0.074 \\
\hline $2 \mathrm{dF} 115901$ & $22.04 \pm 0.08$ & 19.36 & $18.92 \pm 0.04$ & 0.84 & 0.73 & 0.82 & 3.44 & 0.63 & 160.8 \\
\hline iI & $1.07 \pm 0.01$ & -16.67 & -17.11 & 21.09 & 19.79 & 0.54 & $2{ }^{\prime \prime} 6-6{ }^{\prime \prime} 8$ & $0.3 \pm 0.06$ & 0.06 \\
\hline
\end{tabular}

$\dagger$ : morphogical BCD type according to the Loose \& Thuan (1986) classification scheme.

$\mu_{\mathrm{E}, 0}, \alpha$ : central $V$ surface brightness (mag/ $\left.\square^{\prime \prime}\right)$ and exponential scale length (kpc) of the LSB host galaxy.

$m_{\mathrm{LSB}}, M_{\mathrm{LSB}}$ : apparent and absolute $V$ magnitude of the LSB host galaxy (mag).

$m, M$ : total apparent and absolute $V$ magnitude (mag) derived from SBP integration.

$r_{\text {eff }}, \mu_{\text {eff }}: V$ band effective radius (kpc) and mean surface brightness (mag/ $\left.\square^{\prime \prime}\right)$ inside $r_{\text {eff }}$.

$r_{\text {Petr }}, m_{\text {Petr }}$ Petrosian radius $(\mathrm{kpc})$ and apparent magnitude $(\mathrm{mag})$ inside $r_{\text {Petr }}$.

$\log \left(r_{80} / r_{20}\right)$ : light concentration index based on the ratio of the radius $r_{80}$ and $r_{20}$ enclosing, respectively, $80 \%$ and $20 \%$ of the total $V$ light.

$1 / \eta$ : Sérsic exponent of the $V$ SBP.

$r_{\text {eff }} / r_{25}$ : ratio of the isophotal radius at $25 \mathrm{~V}$ mag/ $\square^{\prime \prime}$ and the effective radius.

LSB/total, $(V-I)_{\text {host }}$ : luminosity fraction in the $V$ band and mean $V-I$ colour of the host galaxy.

$D, A_{V}$ : adopted distance in Mpc and $V$ band Galactic absorption in mag. 
Table 4. Emission line intensities.

\begin{tabular}{|c|c|c|c|c|c|c|c|c|}
\hline \multirow[b]{2}{*}{ Ion } & \multicolumn{2}{|c|}{336246} & \multicolumn{2}{|c|}{084585} & \multicolumn{2}{|c|}{169299} & \multicolumn{2}{|c|}{366973} \\
\hline & $F^{1}$ & $E W^{2}$ & $F^{1}$ & $E W^{2}$ & $F^{1}$ & $E W^{2}$ & $F^{1}$ & $E W^{2}$ \\
\hline 3727 [O II] & $114.4 \pm 1.8$ & $99.1 \pm 0.5$ & $44.4 \pm 3.1$ & $62.0 \pm 4.1$ & $64.0 \pm 1.5$ & $47.3 \pm 0.8$ & $45.5 \pm 1.1$ & $56.2 \pm 1.1$ \\
\hline $3750 \mathrm{H} 12$ & $\ldots$ & & $\ldots$ & $\ldots$ & $\ldots$ & $\ldots$ & $1.9 \pm 0.5$ & $2.4 \pm 0.7$ \\
\hline $3771 \mathrm{H} 11$ & $\ldots$ & & $\ldots$ & $\ldots$ & $\ldots$ & & $2.6 \pm 0.6$ & $3.4 \pm 0.8$ \\
\hline 3798 H10 & $3.6 \pm 0.6$ & $3.2 \pm 0.5$ & $\ldots$ & $\ldots$ & $4.6 \pm 1.4$ & $3.4 \pm 1.0$ & $4.3 \pm 0.7$ & $5.8 \pm 0.9$ \\
\hline $3819 \mathrm{He} \mathrm{I}$ & $1.6 \pm 0.6$ & $1.4 \pm 0.5$ & $\ldots$ & $\ldots$ & $\ldots$ & & $0.7 \pm 0.6$ & $1.0 \pm 0.8$ \\
\hline 3835 H9 & $4.4 \pm 0.4$ & $3.8 \pm 0.3$ & $7.3 \pm 2.0$ & $12.3 \pm 3.3$ & $5.2 \pm 1.1$ & $3.8 \pm 0.8$ & $6.2 \pm 0.6$ & $8.6 \pm 0.8$ \\
\hline 3868 [Ne III] & $39.7 \pm 0.7$ & $33.9 \pm 0.4$ & $59.6 \pm 2.6$ & $97.0 \pm 3.6$ & $49.3 \pm 1.3$ & $36.0 \pm 0.8$ & $47.4 \pm 1.0$ & $66.0 \pm 1.0$ \\
\hline $3889 \mathrm{H} 8+\mathrm{He} \mathrm{I}$ & $17.3 \pm 0.4$ & $14.8 \pm 0.3$ & $25.1 \pm 2.2$ & $41.5 \pm 3.5$ & $20.0 \pm 0.9$ & $14.6 \pm 0.6$ & $17.7 \pm 0.7$ & $24.7 \pm 0.8$ \\
\hline $3968[\mathrm{Ne}$ III $]+\mathrm{H} 7$ & $25.5 \pm 0.5$ & $22.0 \pm 0.3$ & $37.0 \pm 2.1$ & $68.2 \pm 3.5$ & $28.1 \pm 1.0$ & $22.0 \pm 0.7$ & $29.0 \pm 0.8$ & $43.2 \pm 0.9$ \\
\hline $4026 \mathrm{He} \mathrm{I}$ & & & $\ldots$ & . & $\ldots$ & & $2.9 \pm 0.6$ & $4.3 \pm 1.0$ \\
\hline $4101 \mathrm{H} \delta$ & $24.9 \pm 0.5$ & $24.8 \pm 0.3$ & $28.5 \pm 1.9$ & $56.9 \pm 3.6$ & $28.4 \pm 0.8$ & $25.0 \pm 0.5$ & $25.5 \pm 0.7$ & $42.4 \pm 0.9$ \\
\hline $4340 \mathrm{H} \gamma$ & $44.5 \pm 0.7$ & $48.2 \pm 0.3$ & $46.8 \pm 1.8$ & $118.6 \pm 3.5$ & $45.0 \pm 1.0$ & $44.4 \pm 0.6$ & $45.2 \pm 10$ & $82.0 \pm 1.2$ \\
\hline 4363 [O III] & $9.9 \pm 0.3$ & $10.7 \pm 0.3$ & $20.4 \pm 1.5$ & $55.1 \pm 3.9$ & $14.5 \pm 0.6$ & $14.5 \pm 0.6$ & $14.4 \pm 0.6$ & $26.3 \pm 1.0$ \\
\hline $4471 \mathrm{He}$ I & $3.4 \pm 0.2$ & $3.9 \pm 0.3$ & $8.2 \pm 1.3$ & $26.0 \pm 3.9$ & $3.4 \pm 0.7$ & $3.6 \pm 0.7$ & $2.6 \pm 0.4$ & $5.2 \pm 0.9$ \\
\hline 4686 He II & $1.1 \pm 0.2$ & $1.4 \pm 0.3$ & $\ldots$ & $\ldots$ & $4.8 \pm 0.6$ & $5.6 \pm 0.6$ & $1.7 \pm 1.1$ & $1.4 \pm 0.9$ \\
\hline 4713 [Ar IV]+He I & $1.1 \pm 0.4$ & $1.4 \pm 0.6$ & $\ldots$ & $\ldots$ & $2.7 \pm 0.6$ & $3.2 \pm 0.7$ & $5.1 \pm 1.0$ & $11.6 \pm 2.4$ \\
\hline $4740[$ Ar IV] & $\ldots$ & & $\ldots$ & $\ldots$ & $2.1 \pm 0.6$ & $2.4 \pm 0.7$ & $3.2 \pm 0.6$ & $7.6 \pm 1.4$ \\
\hline $4861 \mathrm{H} \beta$ & $0.0 \pm 1.5$ & $138.7 \pm 0.5$ & $100.0 \pm 2.9$ & $292.6 \pm 5.2$ & $100.0 \pm 1.8$ & $132.2 \pm 0.9$ & $100.0 \pm 1.8$ & $239.1 \pm 2.0$ \\
\hline III] & $7.9 \pm 2.5$ & $231.1 \pm 0.6$ & $221.0 \pm 5.6$ & $940.8 \pm 9.6$ & $182.9 \pm 3.0$ & $243.9 \pm 1.2$ & $210.6 \pm 3.6$ & $496.4 \pm 2.2$ \\
\hline III] & $503.4 \pm 7.4$ & $698.4 \pm 0.9$ & $665.2 \pm 15.7$ & 2595. \pm 13.6 & $545.2 \pm 8.8$ & $733.4 \pm 1.8$ & $642.4 \pm 10.7$ & 1554. \pm 3.8 \\
\hline 58 & $10.8 \pm 0.3$ & $21.8 \pm 0.6$ & $10.3 \pm 1.3$ & $50.9 \pm 6.1$ & $9.7 \pm 0.6$ & $21.9 \pm 1.4$ & $11.1 \pm 0.6$ & $37.5 \pm 2.1$ \\
\hline 636 & $1.0 \pm 0.4$ & $2.5 \pm 1.0$ & $\ldots$ & $\ldots$ & $\ldots$ & & $\ldots$ & \\
\hline $6563 \mathrm{H} \alpha$ & $296.2 \pm 4.4$ & $753.6 \pm 1.5$ & $237.6 \pm 6.1$ & 1531. \pm 18.1 & $276.8 \pm 4.6$ & $737.0 \pm 3.3$ & $316.0 \pm 5.4$ & 1326. \pm 5.4 \\
\hline $6584[\mathrm{~N}$ I & $4.8 \pm 0.5$ & $12.1 \pm 1.2$ & $\ldots$ & $\ldots$ & $\ldots$ & & . & \\
\hline 6678 & $2.9 \pm 0.4$ & $7.7 \pm 0.9$ & $\ldots$ & $\ldots$ & $4.6 \pm 0.8$ & 2.4 & $3.4 \pm 0.6$ & $15.4 \pm 2.7$ \\
\hline 7065 & $2.4 \pm 0.5$ & $7.7 \pm 1.5$ & $\ldots$ & $\ldots$ & $\ldots$ & $\ldots$ & $4.1 \pm 0.7$ & $21.6 \pm 3.5$ \\
\hline 7136 & $6.0 \pm 0.4$ & $19.3 \pm 1.1$ & $\ldots$ & $\ldots$ & $\ldots$ & $\ldots$ & $5.2 \pm 0.5$ & $28.6 \pm 2.9$ \\
\hline 7320 [O II] & $3.4 \pm 0.5$ & $11.3 \pm 1.6$ & $\ldots$ & ... & $\ldots$ & & $\ldots$ & \\
\hline$C(\mathrm{H} \beta$ & \multirow{2}{*}{\multicolumn{2}{|c|}{$\begin{array}{l}0.060 \pm 0.019 \\
138.60 \pm 0.53\end{array}$}} & \multicolumn{2}{|c|}{$0.000 \pm 0.034$} & \multicolumn{2}{|c|}{$0.000 \pm 0.021$} & \multicolumn{2}{|c|}{$0.165 \pm 0.022$} \\
\hline$F(\mathrm{H} \beta)^{3}$ & & & 7.16 & 0.13 & 35.82 & \pm 0.26 & $92.04=$ & $=0.78$ \\
\hline \multirow[t]{2}{*}{$E W(\mathrm{abs}) \AA$} & \multicolumn{2}{|c|}{$2.2 \pm 0.5$} & \multicolumn{2}{|c|}{$0.0 \pm 2.9$} & \multicolumn{2}{|c|}{$0.5 \pm 1.0$} & \multicolumn{2}{|c|}{$0.4 \pm 0.7$} \\
\hline & \multicolumn{2}{|c|}{211354} & \multicolumn{2}{|c|}{214945} & 301 & 921 & 302 & 61 \\
\hline Ion & $F^{1}$ & $E W^{2}$ & $F^{1}$ & $E W^{2}$ & $F^{1}$ & $E W^{2}$ & $F^{1}$ & $E W^{2}$ \\
\hline 3727 [O II] & $105.8 \pm 1.7$ & $48.6 \pm 0.3$ & $177.6 \pm 2.8$ & $158.6 \pm 0.7$ & $100.1 \pm 1.8$ & $73.8 \pm 0.6$ & $94.3 \pm 1.8$ & $71.4 \pm 0.8$ \\
\hline 38 & $4 \pm 0.4$ & \pm 0.2 & \pm 0.7 & $2.3 \pm 0.6$ & $4.6 \pm 0.7$ & $3.5 \pm 0.5$ & $2.1 \pm 0.7$ & $1.5 \pm 0.5$ \\
\hline 38 & $6 \pm 0.9$ & $24.0 \pm 0.2$ & $37.3 \pm 1.0$ & $0 \pm 0.6$ & $46.6 \pm 1.1$ & $34.5 \pm 0.6$ & $38.1 \pm 1.0$ & $26.8 \pm 0.6$ \\
\hline 388 & $3 \pm 0.5$ & \pm 0.2 & $2 \pm 0.8$ & \pm 0.6 & $14.5 \pm 0.7$ & $10.7 \pm 0.5$ & $12.8 \pm 0.8$ & $9.0 \pm 0.5$ \\
\hline 3968 & $3 \pm 0.6$ & $11.0 \pm 0.2$ & $1 \pm 0.8$ & \pm 0.6 & $24.2 \pm 0.7$ & $18.0 \pm 0.4$ & $19.0 \pm 0.7$ & $13.3 \pm 0.4$ \\
\hline $4101 \mathrm{H} \delta$ & $2 \pm 0.5$ & $9.8 \pm 0.2$ & $18.8 \pm 0.4$ & $14.8 \pm 0.3$ & $22.7 \pm 0.7$ & $16.4 \pm 0.4$ & $20.2 \pm 0.8$ & $15.0 \pm 0.5$ \\
\hline $4340 \mathrm{H} \gamma$ & $43.3 \pm 0.8$ & $23.6 \pm 0.2$ & $38.7 \pm 0.7$ & $32.1 \pm 0.3$ & $45.0 \pm 1.0$ & $36.8 \pm 0.6$ & $39.3 \pm 0.9$ & $29.4 \pm 0.4$ \\
\hline 4363 [O III] & $10.0 \pm 0.4$ & $5.5 \pm 0.2$ & $4.3 \pm 0.3$ & $3.6 \pm 0.3$ & $13.1 \pm 0.8$ & $10.8 \pm 0.6$ & $10.1 \pm 0.5$ & $7.6 \pm 0.4$ \\
\hline $4471 \mathrm{He} \mathrm{I}$ & $4.9 \pm 0.4$ & $2.8 \pm 0.2$ & $4.7 \pm 0.4$ & $4.0 \pm 0.3$ & $4.6 \pm 0.7$ & $3.7 \pm 0.6$ & $3.6 \pm 0.5$ & $2.8 \pm 0.4$ \\
\hline 4713 [Ar IV]+He & & & & & & & $2.7 \pm 0.6$ & $2.2 \pm 0.5$ \\
\hline $4861 \mathrm{H} \beta$ & $.0 \pm 1.6$ & $7 \pm 0.4$ & $.0 \pm 1.6$ & \pm 0.4 & $100.0 \pm 1.8$ & $85.2 \pm 0.6$ & $100.0 \pm 1.7$ & $87.0 \pm 0.6$ \\
\hline 4959 [O III] & $208.3 \pm 3.2$ & $136.9 \pm 0.5$ & $181.1 \pm 2.8$ & $167.2 \pm 0.6$ & $193.6 \pm 3.2$ & $166.1 \pm 0.8$ & $176.8 \pm 2.9$ & $153.2 \pm 0.7$ \\
\hline 5007 [O III] & $626.3 \pm 9.6$ & $414.0 \pm 0.7$ & $545.6 \pm 8.0$ & $505.6 \pm 0.8$ & $579.2 \pm 9.4$ & $502.6 \pm 1.2$ & $531.3 \pm 8.4$ & $467.7 \pm 1.0$ \\
\hline $5876 \mathrm{He} \mathrm{I}$ & $11.6 \pm 0.6$ & $9.9 \pm 0.4$ & $13.6 \pm 0.5$ & $14.8 \pm 0.5$ & $12.0 \pm 0.6$ & $12.5 \pm 0.6$ & $11.2 \pm 0.6$ & $12.2 \pm 0.6$ \\
\hline $6364[\mathrm{O} \mathrm{I}]$ & $\ldots$ & & $2.6 \pm 0.4$ & $3.2 \pm 0.5$ & $1.1 \pm 0.5$ & $1.4 \pm 0.6$ & $\ldots$ & \\
\hline $6563 \mathrm{H} \alpha$ & $322.2 \pm 5.0$ & $309.5 \pm 0.9$ & $377.9 \pm 5.7$ & $474.3 \pm 1.1$ & $341.4 \pm 5.6$ & $409.7 \pm 1.5$ & $352.4 \pm 5.7$ & $438.0 \pm 1.4$ \\
\hline $6678 \mathrm{He} \mathrm{I}$ & $3.5 \pm 0.6$ & $3.6 \pm 0.6$ & $4.5 \pm 0.4$ & $6.3 \pm 0.6$ & $4.6 \pm 0.9$ & $5.7 \pm 1.1$ & $3.6 \pm 0.4$ & $4.5 \pm 0.6$ \\
\hline $7065 \mathrm{He} \mathrm{I}$ & $4 \pm 0.6$ & $5.4 \pm 0.7$ & $3.8 \pm 0.5$ & $5.6 \pm 0.7$ & $3.4 \pm 0.7$ & $4.6 \pm 1.0$ & $\ldots$ & \\
\hline 7136 [Ar III] & $10.1 \pm 0.7$ & $12.4 \pm 0.9$ & $12.7 \pm 0.5$ & $18.1 \pm 0.7$ & $8.8 \pm 0.7$ & $12.6 \pm 1.0$ & $7.0 \pm 0.6$ & $9.7 \pm 0.8$ \\
\hline$C(\mathrm{H} \beta)$ & 0.145 & 0.020 & 0.310 & .020 & 0.265 & $=0.021$ & 0.250 & 0.021 \\
\hline$F(\mathrm{H} \beta)^{3}$ & 94.06 & $=0.52$ & 137.31 & 0.66 & 53.66 & \pm 0.40 & 66.68 & $=0.46$ \\
\hline$E W(\mathrm{abs}) \AA$ & $2.1 \pm$ & 0.2 & 4.4 & 0.6 & 0.2 & $=0.5$ & $4.4 \pm$ & 0.5 \\
\hline
\end{tabular}


P. Papaderos et al.: New southern blue compact dwarf galaxies in the $2 \mathrm{dF}$ Galaxy redshift survey, Online Material p 4

Table 4. continued.

\begin{tabular}{|c|c|c|c|c|c|c|c|c|}
\hline \multirow[b]{2}{*}{ Ion } & \multicolumn{2}{|c|}{ T1304-386 } & \multicolumn{2}{|c|}{ T1304-353 } & \multicolumn{2}{|c|}{ UM 559} & \multicolumn{2}{|c|}{ UM 570} \\
\hline & $F^{1}$ & $E W^{2}$ & $F^{1}$ & $E W^{2}$ & $F^{1}$ & $E W^{2}$ & $F^{1}$ & $E W^{2}$ \\
\hline 3704 H16 & & & & & & & $0.8 \pm 0.3$ & $1.0 \pm 0.4$ \\
\hline 3727 [O II] & $191.0 \pm 3.4$ & $66.9 \pm 2.0$ & $32.6 \pm 0.6$ & $53.4 \pm 0.6$ & $59.7 \pm 0.9$ & $121.0 \pm 0.6$ & $20.6 \pm 0.5$ & $28.2 \pm 0.6$ \\
\hline $3750 \mathrm{H} 12$ & $\ldots$ & $\ldots$ & $1.4 \pm 0.3$ & $2.4 \pm 0.5$ & $\ldots$ & & $1.1 \pm 0.3$ & $1.7 \pm 0.5$ \\
\hline $3771 \mathrm{H} 11$ & $\ldots$ & $\ldots$ & $2.6 \pm 0.4$ & $4.7 \pm 0.6$ & $2.1 \pm 0.2$ & $4.9 \pm 0.5$ & $4.0 \pm 0.4$ & $6.5 \pm 0.6$ \\
\hline 3798 H10 & $\ldots$ & $\ldots$ & $3.9 \pm 0.3$ & $7.3 \pm 0.5$ & $3.5 \pm 0.2$ & $8.3 \pm 0.4$ & $4.9 \pm 0.3$ & $8.2 \pm 0.5$ \\
\hline $3819 \mathrm{He} \mathrm{I}$ & $\ldots$ & $\ldots$ & $\ldots$ & & $\ldots$ & & $0.4 \pm 0.3$ & $0.6 \pm 0.4$ \\
\hline H9 & $\ldots$ & $\ldots$ & $5.7 \pm 0.3$ & $10.9 \pm 0.5$ & $5.5 \pm 0.2$ & $12.8 \pm 0.5$ & $5.6 \pm 0.4$ & $9.2 \pm 0.6$ \\
\hline Je III] & $41.5 \pm 1.4$ & $13.0 \pm 0.4$ & $46.5 \pm 0.7$ & \pm 0.6 & $27.7 \pm 0.5$ & $64.8 \pm 0.6$ & $55.2 \pm 0.9$ & $97.2 \pm 0.8$ \\
\hline 388 & $5 \pm 1.1$ & $3.0 \pm 0.3$ & $16.6 \pm 0.4$ & $32.1 \pm 0.6$ & $15.0 \pm 0.3$ & $35.4 \pm 0.5$ & $18.7 \pm 0.5$ & $33.3 \pm 0.7$ \\
\hline $3968[\mathrm{Ne}$ II & $14.2 \pm 1.0$ & $4.5 \pm 0.3$ & $27.9 \pm 0.5$ & $55.2 \pm 0.6$ & $20.6 \pm 0.4$ & $48.7 \pm 0.4$ & $32.4 \pm 0.6$ & $60.4 \pm 0.7$ \\
\hline $4026 \mathrm{He} \mathrm{I}$ & $\ldots$ & $\ldots$ & $1.4 \pm 0.2$ & $2.8 \pm 0.5$ & $1.5 \pm 0.2$ & $3.6 \pm 0.4$ & $1.6 \pm 0.3$ & $3.1 \pm 0.6$ \\
\hline 4068 & $\ldots$ & $\ldots$ & $\ldots$ & $\ldots$ & $1.0 \pm 0.2$ & $2.4 \pm 0.4$ & $\ldots$ & $\ldots$ \\
\hline $4101 \mathrm{H} \delta$ & $16.2 \pm 1.0$ & $5.4 \pm 0.3$ & $22.1 \pm 0.4$ & $45.1 \pm 0.6$ & $20.5 \pm 0.4$ & $48.4 \pm 0.5$ & $24.4 \pm 0.5$ & $48.7 \pm 0.6$ \\
\hline $4340 \mathrm{H} \gamma$ & $37.8 \pm 1.0$ & $14.3 \pm 0.3$ & $43.6 \pm 0.6$ & $101.8 \pm 0.4$ & $41.3 \pm 0.6$ & $105.1 \pm 0.5$ & $44.7 \pm 0.8$ & $100.8 \pm 0.8$ \\
\hline 4363 [O III] & $7.9 \pm 0.9$ & $3.0 \pm 0.3$ & $17.3 \pm 0.3$ & $40.6 \pm 0.4$ & $9.6 \pm 0.2$ & $24.5 \pm 0.4$ & $20.2 \pm 0.4$ & $45.6 \pm 0.6$ \\
\hline $4388 \mathrm{I}$ & $\ldots$ & $\ldots$ & $0.64 \pm 0.1$ & $1.5 \pm 0.3$ & & & & \\
\hline 4471 & $\ldots$ & $\ldots$ & $3.4 \pm 0.1$ & $8.3 \pm 0.3$ & $3.6 \pm 0.1$ & $9.6 \pm 0.3$ & $4.1 \pm 0.3$ & $10.1 \pm 0.7$ \\
\hline 4640 & $\ldots$ & $\ldots$ & $\ldots$ & $\ldots$ & $0.3 \pm 0.2$ & $0.8 \pm 0.5$ & $\ldots$ & $\ldots$ \\
\hline 465 & $\ldots$ & $\ldots$ & $\ldots$ & $\ldots$ & $0.7 \pm 0.2$ & $1.9 \pm 0.6$ & $\ldots$ & $\ldots$ \\
\hline 468 & $\ldots$ & $\ldots$ & $2.8 \pm 0.1$ & $7.2 \pm 0.3$ & 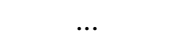 & & $2.1 \pm 0.3$ & $5.5 \pm 0.9$ \\
\hline 47 & $\ldots$ & $\ldots$ & $3.2 \pm 0.1$ & \pm 0.4 & $0.9 \pm 0.1$ & \pm 0.3 & $4.3=$ & $11.2 \pm 0.7$ \\
\hline 47 & $\ldots$ & $\ldots$ & $1 \pm 0.1$ & 0.4 & $0.6 \pm 0.2$ & \pm 0.6 & $3.0 \pm 0.3$ & $8.0 \pm 0.8$ \\
\hline & $100.0 \pm 1.8$ & $45.7 \pm 0.4$ & $100.0 \pm 1.4$ & $281.5 \pm 0.6$ & $100.0 \pm 1.5$ & $293.4 \pm 0.8$ & $100.0 \pm 1.5$ & $290.4 \pm 1.1$ \\
\hline 492 & $\ldots$ & $\ldots$ & $1.1 \pm 0.2$ & $3.0 \pm 0.5$ & $1.1 \pm 0.2$ & $3.3 \pm 0.5$ & $1.6 \pm 0.5$ & $4.8 \pm 1.4$ \\
\hline 4959 & $.8 \pm 2.8$ & \pm 0.4 & $225.9 \pm 3.2$ & $635.9 \pm 0.9$ & $160.7 \pm 2.3$ & $478.2 \pm 0.8$ & $241.6 \pm 3.6$ & $737.2 \pm 1.8$ \\
\hline 5007 [O III & $481.6 \pm 8.0$ & $227.1 \pm 0.7$ & $683.8 \pm 9.8$ & $1923 . \pm 1.6$ & $484.3 \pm 7.0$ & $1405 . \pm 1.5$ & $728.4 \pm 10.7$ & $2258 . \pm 3.1$ \\
\hline & & & & & $0.6 \pm 0.2$ & $2.2 \pm 0.3$ & & \\
\hline $5876 \mathrm{He} \mathrm{I}$ & $13.3 \pm 0.9$ & $7.9 \pm 0.5$ & $12.4 \pm 0.2$ & $47.2 \pm 0.6$ & $11.8 \pm 0.2$ & $49.2 \pm 0.7$ & $10.9 \pm 0.3$ & $48.2 \pm 1.3$ \\
\hline & $\ldots$ & & $0.9 \pm 0.1$ & $3.6 \pm 0.5$ & $0.8 \pm 0.2$ & $3.9 \pm 1.0$ & $\cdots$ & \\
\hline 656 & $364.2 \pm 6.2$ & $50.0 \pm 1.0$ & $335.2 \pm 4.8$ & $1424 . \pm 1.7$ & $340.4 \pm 4.9$ & $1702 . \pm 2.3$ & $303.1 \pm 4.5$ & 1543. \pm 3.7 \\
\hline $6678 \mathrm{He}$ I & $4.9 \pm 1.7$ & $3.4 \pm 1.2$ & $2.9 \pm 0.2$ & $12.6 \pm 0.6$ & $3.3 \pm 0.2$ & $17.2 \pm 0.8$ & & \\
\hline $7065 \mathrm{He} \mathrm{I}$ & $\ldots$ & $\ldots$ & $3.0 \pm 0.1$ & $14.6 \pm 0.6$ & $2.8 \pm 0.2$ & $17.4 \pm 0.9$ & $4.0 \pm 0.4$ & $27.6 \pm 2.8$ \\
\hline 7136 [Ar II & $10.6 \pm 1.6$ & $8.6 \pm 1.3$ & $3.2 \pm 0.1$ & $15.9 \pm 0.6$ & $5.6 \pm 0.2$ & $36.2 \pm 1.1$ & $3.0 \pm 0.3$ & $20.5 \pm 1.9$ \\
\hline $7281 \mathrm{He} \mathrm{I}$ & ... & & $\ldots$ & & $0.9 \pm 0.0$ & $6.0 \pm 2.0$ & $\cdots$ & \\
\hline$C(\mathrm{H} \beta) \mathrm{d}$ & 0.265 & & 0.240 & & 0.240 & & 0.11 & .019 \\
\hline$F(\mathrm{H} \beta)^{3}$ & 55.46 & 0.46 & 395.60 & \pm 0.92 & 357.20 & \pm 0.91 & 172.7 & \pm 0.64 \\
\hline$E W(\mathrm{abs}) \AA$ & 3.2 & 0.3 & $2.6=$ & 0.5 & $5.0 \pm$ & 0.5 & $2.4=$ & 0.5 \\
\hline
\end{tabular}


Table 4. continued.

\begin{tabular}{|c|c|c|c|c|c|c|c|c|}
\hline \multirow[b]{2}{*}{ Ion } & \multicolumn{2}{|c|}{ T1334-326 } & \multicolumn{2}{|c|}{323427} & \multicolumn{2}{|c|}{ T1400-411 } & \multicolumn{2}{|c|}{ T1924-416 } \\
\hline & $F^{1}$ & $E W^{2}$ & $F^{1}$ & $E W^{2}$ & $F^{1}$ & $E W^{2}$ & $F^{1}$ & $E W^{2}$ \\
\hline 3727 [O II] & $117.9 \pm 3.2$ & $99.1 \pm 1.1$ & $103.4 \pm 3.1$ & $49.9 \pm 1.1$ & $94.4 \pm 1.4$ & $105.3 \pm 0.2$ & $124.3 \pm 1.9$ & $55.2 \pm 0.3$ \\
\hline $3750 \mathrm{H} 12$ & $\ldots$ & & $\ldots$ & $\ldots$ & $1.5 \pm 0.1$ & $1.8 \pm 0.1$ & $0.6 \pm 0.2$ & $0.3 \pm 0.1$ \\
\hline $3771 \mathrm{H} 11$ & $\ldots$ & & $\ldots$ & $\ldots$ & $2.8 \pm 0.1$ & $3.4 \pm 0.1$ & $1.4 \pm 1.0$ & $0.6 \pm 0.4$ \\
\hline 3798 H10 & $2.4 \pm 0.8$ & $2.1 \pm 0.7$ & $\ldots$ & $\ldots$ & $4.4 \pm 0.1$ & $5.5 \pm 0.2$ & $3.0 \pm 0.6$ & $1.4 \pm 0.3$ \\
\hline $3819 \mathrm{He}$ I & $\ldots$ & & $\ldots$ & $\ldots$ & $\ldots$ & & $1.8 \pm 0.5$ & $0.8 \pm 0.2$ \\
\hline $3835 \mathrm{H} 9$ & $4.1 \pm 2.3$ & $3.6 \pm 2.0$ & $\ldots$ & & $6.6 \pm 0.2$ & $8.4 \pm 0.1$ & $3.4 \pm 0.5$ & $1.6 \pm 0.2$ \\
\hline 3868 [Ne III] & $45.6 \pm 2.4$ & $41.2 \pm 1.9$ & $31.5 \pm 1.8$ & $12.8 \pm 0.7$ & $47.3 \pm 0.7$ & $60.8 \pm 0.2$ & $44.0 \pm 0.9$ & $20.2 \pm 0.3$ \\
\hline $3889 \mathrm{H} 8+\mathrm{He} \mathrm{I}$ & $14.9 \pm 4.4$ & $13.4 \pm 3.9$ & $9.0 \pm 1.6$ & $3.7 \pm 0.6$ & $20.8 \pm 0.3$ & $26.8 \pm 0.2$ & $14.8 \pm 0.6$ & $6.8 \pm 0.2$ \\
\hline $3968[\mathrm{Ne} \mathrm{III]}+\mathrm{H} 7$ & $24.5 \pm 4.2$ & $22.5 \pm 3.8$ & $13.7 \pm 1.6$ & $5.7 \pm 0.7$ & $28.1 \pm 0.4$ & $37.8 \pm 0.1$ & $24.0 \pm 0.6$ & $11.5 \pm 0.2$ \\
\hline $4026 \mathrm{He} \mathrm{I}$ & $1.7 \pm 0.6$ & $1.6 \pm 0.6$ & $\ldots$ & $\ldots$ & $1.6 \pm 0.1$ & $2.2 \pm 0.1$ & $\ldots$ & $\ldots$ \\
\hline 4068 S II & $0.9 \pm 0.3$ & $0.9 \pm 0.3$ & $\ldots$ & $\ldots$ & $1.0 \pm 0.1$ & $1.4 \pm 0.1$ & $\ldots$ & ... \\
\hline $4101 \mathrm{H} \delta$ & $20.0 \pm 2.8$ & $19.2 \pm 2.7$ & $17.5 \pm 1.6$ & $7.2 \pm 0.6$ & $23.4 \pm 0.4$ & $33.7 \pm 0.2$ & $20.9 \pm 0.4$ & $10.7 \pm 0.2$ \\
\hline $4340 \mathrm{H} \gamma$ & $42.1 \pm 2.1$ & $47.5 \pm 2.0$ & $36.7 \pm 1.4$ & $16.1 \pm 0.6$ & $44.9 \pm 0.6$ & $74.8 \pm 0.2$ & $42.3 \pm 0.7$ & $24.6 \pm 0.2$ \\
\hline 4363 [O III] & $11.2 \pm 2.0$ & $12.7 \pm 2.2$ & $7.3 \pm 1.2$ & $3.0 \pm 0.5$ & $10.2 \pm 0.2$ & $17.2 \pm 0.1$ & $8.7 \pm 0.3$ & $5.1 \pm 0.2$ \\
\hline $4471 \mathrm{He} \mathrm{I}$ & $2.9 \pm 2.1$ & $3.4 \pm 2.5$ & $\ldots$ & $\ldots$ & $3.6 \pm 0.1$ & $6.5 \pm 0.1$ & $2.8 \pm 0.3$ & $1.7 \pm 0.2$ \\
\hline $4658 \mathrm{Fe}$ III & & & $\ldots$ & $\ldots$ & $0.6 \pm 0.1$ & $1.2 \pm 0.1$ & $0.8 \pm 0.2$ & $0.6 \pm 0.2$ \\
\hline $4686 \mathrm{He}$ II & $0.7 \pm 0.3$ & $1.0 \pm 0.4$ & $\ldots$ & $\ldots$ & $1.2 \pm 0.1$ & $2.4 \pm 0.3$ & $1.9 \pm 0.4$ & $1.3 \pm 0.2$ \\
\hline 4713 [Ar IV]+He I & $1.4 \pm 0.7$ & $1.9 \pm 1.0$ & $\ldots$ & $\ldots$ & $1.8 \pm 0.1$ & $3.7 \pm 0.2$ & $1.1 \pm 0.8$ & $0.7 \pm 0.5$ \\
\hline $4740[\mathrm{Ar} \mathrm{IV}]$ & $1.0 \pm 0.3$ & $1.3 \pm 0.4$ & $\ldots$ & $\ldots$ & $1.1 \pm 0.1$ & $2.3 \pm 0.2$ & $0.5 \pm 0.3$ & $0.3 \pm 0.3$ \\
\hline $4861 \mathrm{H} \beta$ & $100.0 \pm 2.4$ & $55.5 \pm 0.8$ & $100.0 \pm 2.9$ & $292.6 \pm 5.2$ & $100.0 \pm 1.4$ & $211.2 \pm 0.3$ & $100.0 \pm 1.5$ & $72.7 \pm 0.2$ \\
\hline $4922 \mathrm{He} \mathrm{I}$ & & & & & $1.2 \pm 0.1$ & $2.5 \pm 0.2$ & $0.6 \pm 0.3$ & $0.5 \pm 0.4$ \\
\hline 4959 [O III] & $213.2 \pm 5.7$ & $310.2 \pm 3.5$ & $142.2 \pm 3.1$ & $79.4 \pm 0.7$ & $204.5 \pm 2.9$ & $441.3 \pm 0.4$ & $187.6 \pm 2.8$ & $138.6 \pm 0.3$ \\
\hline 5007 [O III] & $643.6 \pm 16.1$ & $940.9 \pm 4.8$ & $421.3 \pm 8.6$ & $238.9 \pm 1.1$ & $615.8 \pm 8.8$ & 1339. \pm 0.6 & $563.7 \pm 8.2$ & $420.5 \pm 0.4$ \\
\hline $5199[\mathrm{~N} \mathrm{I}]$ & $\ldots$ & & $\ldots$ & & $0.5 \pm 0.0$ & $1.3 \pm 0.1$ & $0.6 \pm 0.2$ & $0.5 \pm 0.1$ \\
\hline $5876 \mathrm{He} \mathrm{I}$ & $13.1 \pm 1.6$ & $26.6 \pm 3.1$ & $11.1 \pm 1.0$ & $7.8 \pm 0.7$ & $11.8 \pm 0.2$ & $35.6 \pm 0.2$ & $12.3 \pm 0.2$ & $12.7 \pm 0.1$ \\
\hline $6364[\mathrm{O} \mathrm{I}]$ & $2.0 \pm 0.6$ & $4.5 \pm 1.4$ & $\ldots$ & & $0.9 \pm 0.1$ & $2.9 \pm 0.3$ & $1.8 \pm 0.2$ & $2.1 \pm 0.3$ \\
\hline $6563 \mathrm{H} \alpha$ & $350.0 \pm 9.0$ & $798.5 \pm 6.2$ & $343.6 \pm 7.1$ & $276.7 \pm 1.7$ & $335.8 \pm 4.8$ & $1138 . \pm 0.8$ & $348.6 \pm 5.1$ & $419.2 \pm 0.4$ \\
\hline $6678 \mathrm{He} \mathrm{I}$ & $4.1 \pm 0.8$ & $9.5 \pm 2.2$ & $\ldots$ & $\ldots$ & $3.4 \pm 0.1$ & $12.0 \pm 0.3$ & $3.5 \pm 0.1$ & $4.4 \pm 0.1$ \\
\hline $7065 \mathrm{He} \mathrm{I}$ & $2.4 \pm 0.5$ & $7.7 \pm 1.5$ & $\ldots$ & $\ldots$ & $3.5 \pm 0.1$ & $13.8 \pm 0.3$ & $3.0 \pm 0.2$ & $4.0 \pm 0.2$ \\
\hline 7136 [Ar III] & $7.2 \pm 1.3$ & $19.5 \pm 3.5$ & $\ldots$ & $\ldots$ & $8.2 \pm 0.1$ & $34.0 \pm 0.3$ & $5.8 \pm 0.2$ & $8.3 \pm 0.2$ \\
\hline $7320[\mathrm{O} \mathrm{II}]$ & $3.4 \pm 0.5$ & $11.3 \pm 1.6$ & $\ldots$ & $\ldots$ & $0.9 \pm 0.0$ & $3.9 \pm 0.1$ & $\ldots$ & \\
\hline$C(\mathrm{H} \beta) \operatorname{dex}$ & \multicolumn{2}{|c|}{$0.265 \pm 0.033$} & \multicolumn{2}{|c|}{$0.180 \pm 0.027$} & \multicolumn{2}{|c|}{$0.230 \pm 0.018$} & \multicolumn{2}{|c|}{$0.260 \pm 0.019$} \\
\hline$F(\mathrm{H} \beta)^{3}$ & \multicolumn{2}{|c|}{$105.50 \pm 2.11$} & \multicolumn{2}{|c|}{$31.64 \pm 0.44$} & \multicolumn{2}{|c|}{$1705.02 \pm 2.22$} & \multicolumn{2}{|c|}{$973.10 \pm 3.26$} \\
\hline$E W(\mathrm{abs}) \AA$ & \multicolumn{2}{|c|}{$3.0 \pm 0.7$} & \multicolumn{2}{|c|}{$4.7 \pm 0.6$} & \multicolumn{2}{|c|}{$0.4 \pm 0.1$} & \multicolumn{2}{|c|}{$1.2 \pm 0.1$} \\
\hline
\end{tabular}


Table 4. continued.

\begin{tabular}{|c|c|c|c|c|c|c|c|c|}
\hline \multirow[b]{2}{*}{ Ion } & \multicolumn{2}{|c|}{ T2138-405 } & \multicolumn{2}{|c|}{ T2146-391 } & \multicolumn{2}{|c|}{042049} & \multicolumn{2}{|c|}{171716} \\
\hline & $F^{1}$ & $E W^{2}$ & $F^{1}$ & $E W^{2}$ & $F^{1}$ & $E W^{2}$ & $F^{1}$ & $E W^{2}$ \\
\hline $3703 \mathrm{H} 16$ & & & $0.5 \pm 0.2$ & $0.6 \pm 0.3$ & & & & \\
\hline 3727 [O II] & $98.1 \pm 1.5$ & $119.0 \pm 0.6$ & $67.5 \pm 1.0$ & $90.9 \pm 0.4$ & $109.6 \pm 2.4$ & $98.6 \pm 1.2$ & $175.0 \pm 7.3$ & $25.3 \pm 0.7$ \\
\hline $3750 \mathrm{H} 12$ & $1.1 \pm 0.8$ & $1.3 \pm 1.0$ & $1.3 \pm 0.2$ & $1.9 \pm 0.3$ & $\ldots$ & $\ldots$ & $\ldots$ & $\ldots$ \\
\hline $3771 \mathrm{H} 11$ & $2.3 \pm 0.4$ & $3.1 \pm 0.6$ & $3.0 \pm 0.3$ & $4.7 \pm 0.4$ & $\ldots$ & $\ldots$ & $\ldots$ & $\ldots$ \\
\hline 3798 H10 & $5.4 \pm 0.5$ & $7.4 \pm 0.7$ & $4.4 \pm 0.3$ & $7.1 \pm 0.5$ & $\ldots$ & $\ldots$ & $\ldots$ & $\ldots$ \\
\hline $3819 \mathrm{He} \mathrm{I}$ & $1.6 \pm 0.6$ & $2.3 \pm 0.9$ & $0.6 \pm 0.4$ & $1.0 \pm 0.7$ & $\ldots$ & $\ldots$ & $\ldots$ & $\ldots$ \\
\hline 3835 H9 & $5.0 \pm 0.4$ & $7.0 \pm 0.6$ & $6.3 \pm 0.3$ & $10.4 \pm 0.5$ & $\ldots$ & & $\ldots$ & $\ldots$ \\
\hline 3868 [Ne III] & $41.3 \pm 0.8$ & $56.2 \pm 0.6$ & $43.0 \pm 0.7$ & $73.2 \pm 0.5$ & $45.1 \pm 1.3$ & $34.3 \pm 0.7$ & $49.6 \pm 5.4$ & $6.5 \pm 0.7$ \\
\hline $3889 \mathrm{H} 8+\mathrm{He} \mathrm{I}$ & $15.5 \pm 0.5$ & $21.2 \pm 0.5$ & $17.7 \pm 0.4$ & $30.5 \pm 0.5$ & $11.8 \pm 0.8$ & $9.0 \pm 0.6$ & $\ldots$ & $\ldots$ \\
\hline $3968[\mathrm{Ne}$ III $]+\mathrm{H} 7$ & $25.1 \pm 0.5$ & $35.6 \pm 0.5$ & $28.0 \pm 0.5$ & $50.2 \pm 0.5$ & $20.4 \pm 0.9$ & $15.4 \pm 0.6$ & $\ldots$ & $\ldots$ \\
\hline $4026 \mathrm{He} \mathrm{I}$ & $2.1 \pm 0.5$ & $3.1 \pm 0.6$ & $1.6 \pm 0.3$ & $3.0 \pm 0.5$ & $\ldots$ & $\ldots$ & $\ldots$ & $\ldots$ \\
\hline 4068 S III & $2.6 \pm 0.5$ & $3.8 \pm 0.7$ & $1.1 \pm 0.2$ & $2.1 \pm 0.3$ & $\ldots$ & $\ldots$ & $\ldots$ & $\ldots$ \\
\hline $4101 \mathrm{H} \delta$ & $21.1 \pm 0.4$ & $32.6 \pm 0.4$ & $23.4 \pm 0.4$ & $45.3 \pm 0.3$ & $19.6 \pm 0.8$ & $14.6 \pm 0.6$ & $18.6 \pm 3.8$ & $2.7 \pm 0.6$ \\
\hline $4340 \mathrm{H} \gamma$ & $41.2 \pm 0.7$ & $65.3 \pm 0.5$ & $44.3 \pm 0.7$ & $96.9 \pm 0.4$ & $37.4 \pm 1.1$ & $30.4 \pm 0.7$ & $39.3 \pm 3.7$ & $6.2 \pm 0.6$ \\
\hline 4363 [O III] & $8.9 \pm 0.3$ & $14.2 \pm 0.5$ & $12.4 \pm 0.2$ & $27.2 \pm 0.3$ & $8.43 \pm 0.8$ & $6.8 \pm 0.6$ & $5.8 \pm 3.5$ & $1.0 \pm 0.7$ \\
\hline $4388 \mathrm{He} \mathrm{I}$ & $0.6 \pm 0.2$ & $1.0 \pm 0.3$ & $\ldots$ & & $\ldots$ & & $\ldots$ & $\ldots$ \\
\hline $4471 \mathrm{He} \mathrm{I}$ & $3.8 \pm 0.3$ & $6.7 \pm 0.6$ & $3.4 \pm 0.1$ & $8.0 \pm 0.3$ & $5.6 \pm 0.9$ & $3.7 \pm 0.7$ & $\ldots$ & $\ldots$ \\
\hline $4658 \mathrm{Fe}$ III & $1.4 \pm 0.3$ & $2.5 \pm 0.6$ & $0.8 \pm 0.1$ & $2.1 \pm 0.3$ & $\ldots$ & $\ldots$ & $\ldots$ & $\ldots$ \\
\hline $4686 \mathrm{He}$ II & $1.5 \pm 1.2$ & $2.6 \pm 2.1$ & $1.7 \pm 0.1$ & $4.3 \pm 0.3$ & $\ldots$ & $\ldots$ & $\ldots$ & $\ldots$ \\
\hline 4713 [Ar IV]+He I & $1.7 \pm 0.4$ & $3.0 \pm 0.8$ & $2.0 \pm 0.1$ & $5.1 \pm 0.3$ & $\ldots$ & $\ldots$ & $\ldots$ & $\ldots$ \\
\hline 4740 [Ar IV] & $1.4 \pm 0.8$ & $2.7 \pm 1.4$ & $1.2 \pm 0.1$ & $3.2 \pm 0.3$ & $\ldots$ & & $\ldots$ & $\ldots$ \\
\hline $4861 \mathrm{H} \beta$ & $100.0 \pm 1.6$ & $207.9 \pm 0.9$ & $100.0 \pm 1.4$ & $278.7 \pm 0.5$ & $100.0 \pm 2.2$ & $103.8 \pm 1.2$ & $100.0 \pm 4.3$ & $19.4 \pm 0.6$ \\
\hline $4922 \mathrm{He} \mathrm{I}$ & $2.0 \pm 0.5$ & $4.2 \pm 1.1$ & $1.0 \pm 0.1$ & $2.9 \pm 0.4$ & $\ldots$ & & $\ldots$ & $\ldots$ \\
\hline 4959 [O III] & $199.9 \pm 3.0$ & $417.7 \pm 1.1$ & $196.0 \pm 2.8$ & $546.3 \pm 0.7$ & $226.4 \pm 4.4$ & $225.2 \pm 1.4$ & $88.2 \pm 4.0$ & $18.1 \pm 0.6$ \\
\hline 5007 [O III] & $604.1 \pm 9.0$ & 1273. \pm 1.7 & $591.2 \pm 8.4$ & $1651 . \pm 1.0$ & $671.7 \pm 12.6$ & $670.8 \pm 2.2$ & $230.9 \pm 8.2$ & $47.0 \pm 0.7$ \\
\hline 5199 N I & $0.7 \pm 0.3$ & $1.7 \pm 0.7$ & $0.7 \pm 0.1$ & $2.2 \pm 0.4$ & $\ldots$ & $\ldots$ & $\ldots$ & $\ldots$ \\
\hline $5270 \mathrm{Fe}$ III & $1.6 \pm 0.5$ & $4.0 \pm 1.3$ & $\ldots$ & $\ldots$ & $\ldots$ & $\ldots$ & $\ldots$ & $\ldots$ \\
\hline $5518 \mathrm{Cl}$ III & $1.2 \pm 0.4$ & $3.1 \pm 1.1$ & $\ldots$ & $\ldots$ & $\ldots$ & $\ldots$ & $\ldots$ & $\ldots$ \\
\hline $5538 \mathrm{Cl}$ III & $0.9 \pm 0.3$ & $2.4 \pm 0.8$ & $\ldots$ & & $\ldots$ & & $\ldots$ & $\ldots$ \\
\hline $5876 \mathrm{He} \mathrm{I}$ & $13.6 \pm 0.4$ & $40.2 \pm 1.1$ & $11.2 \pm 0.2$ & $45.5 \pm 0.5$ & $12.8 \pm 0.8$ & $17.0 \pm 1.0$ & $15.4 \pm 4.0$ & $4.3 \pm 1.1$ \\
\hline $6364[\mathrm{O}$ I] & $1.5 \pm 0.3$ & $5.2 \pm 1.1$ & $1.3 \pm 0.2$ & $6.2 \pm 0.8$ & $\ldots$ & & $\ldots$ & $\ldots$ \\
\hline $6563 \mathrm{H} \alpha$ & $331.8 \pm 5.0$ & 1192. \pm 2.4 & $324.5 \pm 4.6$ & $1547 . \pm 1.6$ & $335.8 \pm 6.5$ & $507.2 \pm 2.7$ & $312.9 \pm 10.9$ & $107.6 \pm 1.5$ \\
\hline 6584 [N II] & $10.7 \pm 0.9$ & $61.8 \pm 2.9$ & $\ldots$ & & $10.0 \pm 3.2$ & $15.1 \pm 4.9$ & $\ldots$ & $\ldots$ \\
\hline $6678 \mathrm{He} \mathrm{I}$ & $3.9 \pm 0.3$ & $14.5 \pm 1.3$ & $2.0 \pm 0.1$ & $10.7 \pm 0.6$ & $4.9 \pm 0.9$ & $8.7 \pm 1.6$ & $\ldots$ & $\ldots$ \\
\hline $7065 \mathrm{He}$ I & $\ldots$ & $\ldots$ & $3.2 \pm 0.1$ & $19.1 \pm 0.7$ & & & $\ldots$ & ... \\
\hline 7136 [Ar III] & & & $10.2 \pm 0.2$ & $60.4 \pm 0.7$ & $11.9 \pm 1.4$ & $18.9 \pm 0.2$ & $\ldots$ & ... \\
\hline$C(\mathrm{H} \beta) \operatorname{dex}$ & \multicolumn{2}{|c|}{$0.185 \pm 0.019$} & \multirow{2}{*}{\multicolumn{2}{|c|}{$\begin{array}{l}0.195 \pm 0.019 \\
460.09 \pm 0.89\end{array}$}} & \multirow{3}{*}{\multicolumn{2}{|c|}{$\begin{array}{c}0.165 \pm 0.025 \\
82.49 \pm 0.99 \\
5.9 \pm 0.6\end{array}$}} & \multirow{3}{*}{\multicolumn{2}{|c|}{$\begin{aligned} 0.080 & \pm 0.045 \\
6.61 & \pm 0.19 \\
1.5 & \pm 0.4\end{aligned}$}} \\
\hline$F(\mathrm{H} \beta)^{3}$ & 269.50 & \pm 1.19 & & & & & & \\
\hline$E W(\mathrm{abs}) \AA$ & 5.4 & $=1.0$ & 0.8 & 0.3 & & & & \\
\hline
\end{tabular}


Table 4. continued.

\begin{tabular}{|c|c|c|c|c|c|c|c|c|}
\hline \multirow[b]{2}{*}{ Ion } & \multicolumn{2}{|c|}{$171716 b+c$} & \multicolumn{2}{|c|}{116230} & \multicolumn{2}{|c|}{181442} & \multicolumn{2}{|c|}{115901} \\
\hline & $F^{1}$ & $E W^{2}$ & $F^{1}$ & $E W^{2}$ & $F^{1}$ & $E W^{2}$ & $F^{1}$ & $E W^{2}$ \\
\hline 3727 [O II] & $124.7 \pm 3.8$ & $41.6 \pm 1.0$ & $109.8 \pm 2.3$ & $79.1 \pm 0.9$ & $134.5 \pm 7.0$ & $65.4 \pm 2.7$ & $34.0 \pm 0.6$ & $65.6 \pm 0.7$ \\
\hline $3750 \mathrm{H} 12$ & $\ldots$ & $\ldots$ & $\ldots$ & $\ldots$ & $\ldots$ & $\ldots$ & $2.2 \pm 0.2$ & $4.8 \pm 0.4$ \\
\hline $3771 \mathrm{H} 11$ & $\ldots$ & $\ldots$ & $\ldots$ & $\ldots$ & $\ldots$ & $\ldots$ & $3.9 \pm 0.2$ & $8.9 \pm 0.5$ \\
\hline 3798 H10 & $\ldots$ & $\ldots$ & $3.6 \pm 1.9$ & $2.5 \pm 1.3$ & $\ldots$ & $\ldots$ & $4.4 \pm 0.2$ & $10.5 \pm 0.5$ \\
\hline 3835 H9 & $\ldots$ & $\ldots$ & $4.0 \pm 1.5$ & $2.7 \pm 1.0$ & $\ldots$ & & $7.6 \pm 0.3$ & $18.7 \pm 0.6$ \\
\hline 3868 [Ne III] & $31.0 \pm 2.7$ & $9.1 \pm 0.9$ & $52.2 \pm 1.5$ & $34.2 \pm 0.8$ & $40.6 \pm 4.1$ & $20.7 \pm 2.0$ & $33.1 \pm 0.6$ & $82.0 \pm 0.8$ \\
\hline $3889 \mathrm{H} 8+$ & $13.4 \pm 2.5$ & $3.9 \pm 0.7$ & $16.4 \pm 1.0$ & $10.6 \pm 0.6$ & $18.1 \pm 5.3$ & $9.4 \pm 2.8$ & $18.7 \pm 0.4$ & $47.4 \pm 0.7$ \\
\hline $3968[\mathrm{Ne}$ III $]+\mathrm{H} 7$ & $12.2 \pm 2.0$ & $3.7 \pm 0.7$ & $25.4 \pm 1.0$ & $16.3 \pm 0.6$ & $25.6 \pm 4.1$ & $14.2 \pm 2.2$ & $25.8 \pm 0.5$ & $67.8 \pm 0.7$ \\
\hline $4026 \mathrm{He} \mathrm{I}$ & $\ldots$ & & $\ldots$ & $\ldots$ & $\ldots$ & $\ldots$ & $1.8 \pm 0.3$ & $4.7 \pm 0.7$ \\
\hline $4101 \mathrm{H} \delta$ & $16.9 \pm 1.6$ & $5.2 \pm 0.5$ & $18.7 \pm 0.9$ & $11.8 \pm 0.6$ & $25.0 \pm 3.2$ & $15.0 \pm 1.8$ & $24.1 \pm 0.4$ & $67.6 \pm 0.7$ \\
\hline $4340 \mathrm{H} \gamma$ & $34.5 \pm 1.4$ & $11.9 \pm 0.5$ & $42.2 \pm 1.2$ & $29.6 \pm 0.6$ & $47.3 \pm 3.0$ & $28.7 \pm 1.6$ & $45.5 \pm 0.8$ & $138.5 \pm 1.1$ \\
\hline 4363 [O III] & $7.2 \pm 0.9$ & $1.9 \pm 0.4$ & $10.6 \pm 0.9$ & $7.2 \pm 0.6$ & $6.4 \pm 2.0$ & $4.0 \pm 1.2$ & $12.7 \pm 0.3$ & $38.8 \pm 0.8$ \\
\hline $4471 \mathrm{He} \mathrm{I}$ & $\ldots$ & $\ldots$ & $5.1 \pm 0.9$ & $3.3 \pm 0.6$ & $\ldots$ & $\ldots$ & $4.0 \pm 0.2$ & $13.3 \pm 0.7$ \\
\hline $4658 \mathrm{Fe}$ III & $\ldots$ & $\ldots$ & $\ldots$ & $\ldots$ & $\ldots$ & $\ldots$ & $0.7 \pm 0.2$ & $2.8 \pm 0.8$ \\
\hline $4686 \mathrm{He}$ II & $2.8 \pm 0.6$ & $1.4 \pm 0.3$ & $\ldots$ & $\ldots$ & $\ldots$ & $\ldots$ & $1.5 \pm 0.2$ & $5.6 \pm 0.7$ \\
\hline 4713 [Ar IV] + He I & $\ldots$ & $\ldots$ & $\ldots$ & $\ldots$ & $\ldots$ & $\ldots$ & $2.0 \pm 0.2$ & $7.3 \pm 0.8$ \\
\hline 4740 [Ar IV] & $\ldots$ & $\ldots$ & $\ldots$ & $\ldots$ & $\ldots$ & $\ldots$ & $1.0 \pm 0.2$ & $3.9 \pm 0.8$ \\
\hline $4861 \mathrm{H} \beta$ & $100.0 \pm 2.7$ & $48.8 \pm 1.0$ & $100.0 \pm 2.0$ & $85.7 \pm 0.9$ & $100.0 \pm 4.3$ & $79.0 \pm 2.2$ & $100.0 \pm 1.6$ & $384.1 \pm 1.7$ \\
\hline $4922 \mathrm{He} \mathrm{I}$ & $\ldots$ & & $\ldots$ & & $\ldots$ & & $1.1 \pm 0.2$ & $4.4 \pm 0.9$ \\
\hline 4959 [O III] & $91.5 \pm 2.6$ & $46.1 \pm 0.9$ & $196.5 \pm 3.7$ & $153.6 \pm 1.0$ & $156.2 \pm 5.8$ & $129.1 \pm 2.4$ & $158.7 \pm 2.4$ & $662.9 \pm 2.3$ \\
\hline 5007 [O III] & $7.2 \pm 6.2$ & $136.9 \pm 1.1$ & $592.8 \pm 10.6$ & $466.8 \pm 1.5$ & $481.8 \pm 16.0$ & $416.0 \pm 3.7$ & $476.3 \pm 7.1$ & $2036 . \pm 3.6$ \\
\hline $5876 \mathrm{He} \mathrm{I}$ & $10.3 \pm 4.2$ & $7.4 \pm 0.9$ & $12.0 \pm 1.2$ & $11.6 \pm 0.7$ & $\ldots$ & & $11.6 \pm 0.3$ & $79.6 \pm 2.0$ \\
\hline $6563 \mathrm{H} \alpha$ & $312.8 \pm 7.2$ & $280.5 \pm 2.9$ & $310.0 \pm 5.7$ & $353.5 \pm 1.8$ & $321.8 \pm 11.5$ & $380.7 \pm 6.0$ & $304.6 \pm 4.6$ & $2255 . \pm 5.7$ \\
\hline $6678 \mathrm{He} \mathrm{I}$ & $\ldots$ & $\ldots$ & $3.6 \pm 1.0$ & $4.3 \pm 0.3$ & $\ldots$ & $\ldots$ & $3.2 \pm 0.2$ & $29.2 \pm 2.1$ \\
\hline $7065 \mathrm{He} \mathrm{I}$ & $\ldots$ & $\ldots$ & $\ldots$ & $\ldots$ & $\ldots$ & $\ldots$ & $6.4 \pm 0.3$ & $57.8 \pm 2.9$ \\
\hline 7136 [ & $\ldots$ & $\ldots$ & $6.4 \pm 0.9$ & $8.0 \pm 1.1$ & $\ldots$ & $\ldots$ & $\ldots$ & $\ldots$ \\
\hline 7320 [O II] & $\ldots$ & $\ldots$ & $3.4 \pm 0.5$ & $11.3 \pm 1.6$ & $\ldots$ & $\ldots$ & $\ldots$ & \\
\hline$C(\mathrm{H} \beta) \operatorname{dex}$ & $0.060=$ & 0.030 & $0.080 \pm$ & 0.024 & $0.165 \pm$ & 0.046 & 0.125 & 0.020 \\
\hline$F(\mathrm{H} \beta)^{3}$ & 15.95 & 0.26 & 23.06 & 0.24 & $24.66 \pm$ & 0.71 & 70.15 & $=0.32$ \\
\hline$E W($ abs $) \AA$ & $4.9=$ & 0.5 & $4.7 \pm$ & 1.2 & $0.2 \pm$ & 1.8 & 1.2 & 0.4 \\
\hline
\end{tabular}

${ }^{1} F=F(\lambda) / F(\mathrm{H} \beta)$.

2 in $\AA$.

${ }^{3}$ in units $10^{-16} \mathrm{erg} \mathrm{s}^{-1} \mathrm{~cm}^{-2}$. 
P. Papaderos et al.: New southern blue compact dwarf galaxies in the $2 \mathrm{dF}$ Galaxy redshift survey, Online Material p 8

Table 5. Heavy element abundances.

\begin{tabular}{|c|c|c|c|c|c|c|c|c|}
\hline Property & 336246 & 084585 & 169299 & 366973 & 211354 & 214945 & 301921 & 302461 \\
\hline$t_{\mathrm{e}}(\mathrm{O}$ III $)(\mathrm{K})^{5}$ & $1.53 \pm 0.02$ & $1.90 \pm 0.08$ & $1.75 \pm 0.04$ & $1.66 \pm 0.04$ & $1.41 \pm 0.02$ & $1.11 \pm 0.03$ & $1.69 \pm 0.06$ & $1.55 \pm 0.04$ \\
\hline$t_{\mathrm{e}}(\mathrm{O}$ II $)(\mathrm{K})^{5}$ & $1.39 \pm 0.02$ & $1.54 \pm 0.06$ & $1.48 \pm 0.03$ & $1.45 \pm 0.03$ & $1.33 \pm 0.04$ & $1.16 \pm 0.03$ & $1.46 \pm 0.05$ & $1.40 \pm 0.04$ \\
\hline $\mathrm{O}^{+} / \mathrm{H}^{+}(\rangle$ & $1.29 \pm 0.06$ & $0.36 \pm 0.04$ & $0.57 \pm 0.04$ & $0.49 \pm 0.03$ & $1.42 \pm 0.07$ & $4.30 \pm 0.37$ & $1.13 \pm 0.10$ & $1.15 \pm 0.09$ \\
\hline $\mathrm{O}^{++} / \mathrm{H}^{+}\left(\times 10^{5}\right)$ & $5.14 \pm 0.20$ & $4.26 \pm 0.43$ & $4.12 \pm 0.24$ & $5.41 \pm$ & $7.57 \pm 0.36$ & $12.82 \pm 1.13$ & $4.62 \pm 0.38$ & $4.96 \pm 0.35$ \\
\hline $\mathrm{O}^{+3} / \mathrm{H}^{+}($ & $0.08 \pm 0.02$ & $\ldots$ & $0.15 \pm 0.03$ & $0.10 \pm 0.07$ & $\ldots$ & $\ldots$ & $\ldots$ & $\ldots$ \\
\hline $\mathrm{O} / \mathrm{H}\left(\times 10^{5}\right)$ & $6.52 \pm 0.21$ & $4.62 \pm 0.43$ & $4.84 \pm$ & 6.00 & $8.99 \pm 0$ & $17.11 \pm$ & $5.75 \pm 0.39$ & $6.11 \pm 0.36$ \\
\hline $\mathrm{O} / \mathrm{H})$ & $7.81 \pm 0.01$ & $7.66 \pm 0.04$ & $7.68 \pm 0$ & $7.78=$ & $7.95 \pm 0.02$ & $8.23=$ & $7.76 \pm 0.03$ & $7.79 \pm 0.02$ \\
\hline$[\mathrm{O} / \mathrm{H}]$ & $-0.85 \pm 0.01$ & $-1.00 \pm 0.04$ & $-0.98 \pm 0.02$ & $-0.88 \pm$ & $-0.71 \pm 0.02$ & $-0.43=$ & $-0.90 \pm 0.03$ & $-0.87 \pm 0.02$ \\
\hline $\mathrm{Ne}^{++} / \mathrm{H}$ & $0.95 \pm$ & $0.79 \pm 0.08$ & $0.78 \pm 0.05$ & $0.96 \pm 0.06$ & $1.61 \pm 0.08$ & $2.82 \pm$ & $0.95 \pm 0.08$ & $0.93 \pm 0.07$ \\
\hline & 1.27 & 1.08 & 1.18 & 1.11 & 1.19 & 1.34 & 1.24 & 1.23 \\
\hline $\log (1$ & $-0.74 \pm 0.02$ & $0.73 \pm 0.06$ & $-0.72 \pm 0.04$ & $-0.75=$ & $-0.67 \pm 0.03$ & $-0.66 \pm 0.05$ & $-0.69 \pm 0.05$ & $-0.73 \pm 0.04$ \\
\hline$[\mathrm{Ne} / \mathrm{O}$ & $0.08 \pm 0.02$ & $0.09 \pm 0.06$ & $0.10 \pm 0.04$ & $0.07=$ & $0.15 \pm 0.03$ & $0.16 \pm$ & $0.13 \pm 0.05$ & $0.09 \pm 0.04$ \\
\hline$\left(\times 10^{7}\right)$ & $2.17 \pm 0.14$ & $\cdots$ & & 0.16 & $3.73 \pm 0.30$ & $5.49 \pm 0.34$ & $2.26 \pm 0.21$ & $1.97 \pm 0.18$ \\
\hline$\left.<10^{7}\right)$ & & $\ldots$ & $2.11 \pm 0.61$ & 3.75 & & & & \\
\hline & 1.91 & $\ldots$ & 2.14 & 1.01 & 2.15 & 1.71 & 1.92 & 1.97 \\
\hline & $-2.20 \pm 0.03$ & $\ldots$ & $-2.03 \pm$ & $-2.05 \pm 0.06$ & $-2.05 \pm 0.04$ & $-2.26 \pm$ & $-2.12 \pm 0.05$ & $-2.20 \pm 0.05$ \\
\hline$[\mathrm{Ar} / \mathrm{O}$ & $0.28 \pm 0.03$ & ... & $0.45 \pm 0.13$ & 0.43 & $0.43 \pm 0.04$ & $0.22 \pm 0.04$ & $0.36 \pm 0.05$ & $0.28 \pm 0.05$ \\
\hline Property & T1304-386 & T1304-353 & UM 559 & UM 570 & T1334-326 & 323427 & T1400-411 & T1924-416 \\
\hline$\overline{t_{\mathrm{e}}(\mathrm{O} \mathrm{I}}$ & $1.46 \pm$ & $.79 \pm 0.02$ & $1.58 \pm$ & 02 & $1.49 \pm 0.12$ & $1.47=$ & $1.45 \pm 0.01$ & $1.42 \pm 0.02$ \\
\hline$t_{\mathrm{e}}(\mathrm{O} \mathrm{II}$ & $1.35 \pm$ & $50 \pm($ & $1.41 \pm$ & 1.51 & $1.37 \pm$ & 1.36 & $1.35 \pm$ & 1.34 \\
\hline $\mathrm{O}^{+} /$ & $2.56 \pm$ & $3 \pm$ & $0.72 \pm$ & 0.19 & $1.59 \pm$ & $1.27=$ & $1.33 \pm$ & $1.83 \pm 0.09$ \\
\hline $\left.0^{5}\right)$ & $5.16 \pm 0.74$ & $4.80 \pm 0.14$ & $4.46 \pm 0$ & $4.92 \pm 0.16$ & $6.77 \pm$ & $4.42 \pm$ & $7.14 \pm$ & $6.80 \pm$ \\
\hline$\left.\times 10^{5}\right)$ & $\ldots$ & $0.19 \pm 0.01$ & ... & $\ldots$ & $0.06 \pm$ & 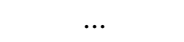 & $0.12 \pm 0.01$ & $0.19 \pm 0.04$ \\
\hline $\mathrm{O} /$ & \pm & $2 \pm 0$ & \pm & 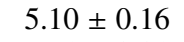 & 8. & 5 . & $3 \pm$ & $8.82=$ \\
\hline $\mathrm{O} / \mathrm{H})$ & $7.89 \pm 0.05$ & $7.73 \pm 0$ & $7.72 \pm$ & & $7.92 \pm$ & $7.76=$ & $7.93 \pm$ & $7.94 \pm 0.02$ \\
\hline$[\mathrm{O} / \mathrm{H}$ & $-0.77 \pm 0.05$ & $-0.93 \pm 0.01$ & $-0.94 \pm($ & $-0.95 \pm$ & $-0.74 \pm$ & $-0.90 \pm$ & $-0.73 \pm 0.01$ & $-0.72 \pm 0.02$ \\
\hline $\mathrm{Ne}^{++} / \mathrm{H}^{+}$ & $1.21 \pm$ & $0.81 \pm$ & $0.66 \pm$ & 0.84 & $1.29=$ & 0.84 & $1.46=$ & 1.45 \\
\hline & & & & & & 1 & 1.2 & 1.3 \\
\hline $\log (\mathrm{Ne} / \mathrm{O})$ & $-0.63 \pm 0.08$ & $-0.77 \pm 0.02$ & $-0.83 \pm 0.02$ & $-0.77 \pm 0.02$ & $-0.72 \pm 0.12$ & $-0.72 \pm$ & $-0.69 \pm 0.02$ & $-0.67 \pm 0.03$ \\
\hline$[\mathrm{Ne} / \mathrm{O}]^{4}$ & $0.19 \pm 0.08$ & $0.05 \pm 0.02$ & $-0.01 \pm$ & $0.05 \pm 0.02$ & $0.10 \pm 0.12$ & $0.10 \pm$ & $0.13 \pm 0.02$ & $0.15 \pm 0.03$ \\
\hline $\left.0^{7}\right)$ & $3.20 \pm 0.56$ & $78 \pm 0.04$ & 06 & .08 & 2. & & $2.74 \pm 0.06$ & $1.92 \pm 0.07$ \\
\hline $\mathrm{Ar}$ & $\cdots$ & $6 \pm 0$ & 0.8 & $2.79 \pm 0.28$ & $1.40 \pm 0$ & $\cdots$ & $1.82 \pm 0.15$ & $0.83 \pm 0.51$ \\
\hline $\mathrm{ICF}(\mathrm{A}$ & & & & $1 .($ & 1.03 & . & 1.02 & 1.04 \\
\hline $\log (\mathrm{Ar} / \mathrm{O}$ & $-2.20 \pm 0.09$ & $-2.27 \pm$ & $-2.32 \pm$ & $-2.15 \pm$ & $-2.36 \pm$ & $\ldots$ & $-2.26 \pm$ & $-2.49 \pm 0.08$ \\
\hline$[\mathrm{Ar} / \mathrm{O}]^{4}$ & $0.28 \pm 0.09$ & $0.21 \pm 0.03$ & $0.16 \pm 0.05$ & $0.33 \pm 0.06$ & $0.12 \pm 0.12$ & ... & $0.22 \pm 0.02$ & $-0.01 \pm 0.08$ \\
\hline$\left(\times 10^{7}\right)$ & $\cdots$ & $\cdots$ & $.55 \pm$ & $\cdots$ & & & $1.61 \pm 0.18$ & $2.19 \pm 0.64$ \\
\hline & $\ldots$ & $\ldots$ & 8.98 & ... & $\ldots$ & .. & 8.08 & 6.02 \\
\hline & $\ldots$ & $\ldots$ & $-1.57 \pm$ & ... & ... & 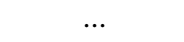 & $-1.82 \pm$ & $-1.82 \pm 0.13$ \\
\hline$[\mathrm{O} / \mathrm{Fe}]^{4}$ & $\ldots$ & - & $-0.36 \pm 0.15$ & $\ldots$ & - & $\cdots$ & $-0.61 \pm 0.05$ & $-0.61 \pm 0.13$ \\
\hline
\end{tabular}


P. Papaderos et al.: New southern blue compact dwarf galaxies in the $2 \mathrm{dF}$ Galaxy redshift survey, Online Material p 9

Table 5. continued.

\begin{tabular}{|c|c|c|c|c|c|c|c|c|}
\hline Property & T2138-405 & $\overline{T 2146-391}$ & $\overline{042049}$ & $\overline{171716}$ & $171716 \mathrm{~b}+\mathrm{c}$ & 116230 & 181442 & $\overline{115901}$ \\
\hline$t_{\mathrm{e}}(\mathrm{O}$ III $)(\mathrm{K})^{5}$ & $1.38 \pm 0.02$ & $1.61 \pm 0.02$ & $1.28 \pm 0.05$ & $1.69 \pm 0.57$ & $1.78 \pm 0.01$ & $1.47 \pm 0.06$ & $1.32 \pm 0.02$ & $1.80 \pm 0.03$ \\
\hline$t_{\mathrm{e}}(\mathrm{O}$ II $)(\mathrm{K})^{5}$ & $1.31 \pm 0.02$ & $1.42 \pm 0.01$ & $1.26 \pm 0.04$ & $1.46 \pm 0.46$ & $1.50 \pm 0.11$ & $1.36 \pm 0.05$ & $1.28 \pm 0.02$ & $1.50 \pm 0.02$ \\
\hline $\mathrm{O}^{+} / \mathrm{H}^{+}\left(\times 10^{5}\right)$ & $1.44 \pm 0.07$ & $0.78 \pm 0.02$ & $1.75 \pm 0.20$ & $1.62 \pm 1.38$ & $1.03 \pm 0.19$ & $1.29 \pm 0.14$ & $2.15 \pm 0.78$ & $0.32 \pm 0.01$ \\
\hline $\mathrm{O}^{++} / \mathrm{H}^{+}\left(\times 10^{5}\right)$ & $7.87 \pm 0.37$ & $5.34 \pm 0.15$ & $10.31 \pm 1.13$ & $1.82 \pm 1.49$ & $1.78 \pm 0.32$ & $6.40 \pm 0.68$ & $7.17 \pm 2.53$ & $3.36 \pm 0.13$ \\
\hline $\mathrm{O}^{+3} / \mathrm{H}^{+}\left(\times 10^{5}\right)$ & $0.13 \pm 0.11$ & $0.19 \pm 0.02$ & $\ldots$ & $\ldots$ & $\ldots$ & $\ldots$ & $\ldots$ & $0.06 \pm 0.01$ \\
\hline $\mathrm{O} / \mathrm{H}\left(\times 10^{5}\right)$ & $9.44 \pm 0.40$ & $6.31 \pm 0.16$ & $12.06 \pm 1.14$ & $3.44 \pm 2.03$ & $2.81 \pm 0.37$ & $7.70 \pm 0.69$ & $9.32 \pm 2.65$ & $3.74 \pm 0.13$ \\
\hline $12+\log (\mathrm{O} / \mathrm{H})$ & $7.98 \pm 0.02$ & $7.80 \pm 0.01$ & $8.08 \pm 0.04$ & $7.54 \pm 0.26$ & $7.45 \pm 0.06$ & $7.89 \pm 0.04$ & $7.97 \pm 0.12$ & $7.57 \pm 0.02$ \\
\hline$[\mathrm{O} / \mathrm{H}]^{4}$ & $-0.68 \pm 0.02$ & $-0.86 \pm 0.01$ & $-0.58 \pm 0.04$ & $-1.12 \pm 0.26$ & $-1.21 \pm 0.06$ & $-0.77 \pm 0.04$ & $-0.69 \pm 0.12$ & $-1.09 \pm 0.02$ \\
\hline $\mathrm{Ne}^{++} / \mathrm{H}^{+}\left(\times 10^{5}\right)$ & $1.42 \pm 0.07$ & $0.97 \pm 0.03$ & $1.86 \pm 0.22$ & $0.85 \pm 0.69$ & $0.45 \pm 0.09$ & $1.35 \pm 0.15$ & $1.61 \pm 0.63$ & $0.53 \pm 0.02$ \\
\hline $\mathrm{ICF}(\mathrm{Ne})$ & 1.20 & 1.18 & 1.17 & 1.89 & 1.58 & 1.20 & 1.30 & 1.11 \\
\hline $\log (\mathrm{Ne} / \mathrm{O})$ & $-0.74 \pm 0.03$ & $-0.74 \pm 0.02$ & $-0.74 \pm 0.07$ & $-0.33 \pm 0.44$ & $-0.60 \pm 0.10$ & $-0.68 \pm 0.06$ & $-0.65 \pm 0.21$ & $-0.80 \pm 0.02$ \\
\hline$[\mathrm{Ne} / \mathrm{O}]^{4}$ & $0.08 \pm 0.03$ & $0.08 \pm 0.02$ & $0.08 \pm 0.07$ & $0.49 \pm 0.44$ & $0.22 \pm 0.10$ & $0.14 \pm 0.06$ & $0.17 \pm 0.21$ & $0.02 \pm 0.02$ \\
\hline $\mathrm{Ar}^{++} / \mathrm{H}^{+}\left(\times 10^{7}\right)$ & $\ldots$ & $3.02 \pm 0.07$ & $4.65 \pm 0.65$ & $\ldots$ & $\cdots$ & $2.33 \pm 0.37$ &.. & \\
\hline $\mathrm{Ar}^{+3} / \mathrm{H}^{+}\left(\times 10^{7}\right)$ & $2.54 \pm 1.36$ & $1.52 \pm 0.13$ & $x_{0}=-1$ & $\ldots$ & $\ldots$ & $\ldots$ & $\ldots$ & $1.03 \pm 0.20$ \\
\hline $\mathrm{ICF}(\mathrm{Ar})$ & 2.31 & 1.01 & 2.25 & $\ldots$ & $\ldots$ & 2.09 & $\ldots$ & 1.69 \\
\hline $\log (\mathrm{Ar} / \mathrm{O})$ & $-2.21 \pm 0.23$ & $-2.14 \pm 0.02$ & $-2.06 \pm 0.07$ & $\ldots$ & $\ldots$ & $-2.20 \pm 0.08$ & $\ldots$ & $-2.33 \pm 0.08$ \\
\hline$[\mathrm{Ar} / \mathrm{O}]^{4}$ & $0.27 \pm 0.23$ & $0.34 \pm 0.02$ & $0.42 \pm 0.07$ & $\ldots$ & $\ldots$ & $0.28 \pm 0.08$ & $\ldots$ & $0.15 \pm 0.08$ \\
\hline $\mathrm{Fe}^{++} / \mathrm{H}^{+}\left(\times 10^{7}\right)$ & $3.78 \pm 0.95$ & $1.79 \pm 0.29$ & $\ldots$ & $\ldots$ & $\ldots$ & $\ldots$ & $\cdots$ & $1.42 \pm 0.41$ \\
\hline $\mathrm{ICF}(\mathrm{Fe})$ & 8.22 & 10.08 & $\ldots$ & $\ldots$ & $\ldots$ & $\ldots$ & $\ldots$ & 14.65 \\
\hline $\log (\mathrm{Fe} / \mathrm{O})$ & $-1.48 \pm 0.11$ & $-1.54 \pm 0.07$ & $\ldots$ & $\ldots$ & $\ldots$ & $\ldots$ & $\ldots$ & $-1.26 \pm 0.13$ \\
\hline$[\mathrm{O} / \mathrm{Fe}]^{4}$ & $-0.27 \pm 0.11$ & $-0.33 \pm 0.07$ & $\ldots$ & $\ldots$ & $\ldots$ & $\ldots$ & $\ldots$ & $-0.05 \pm 0.13$ \\
\hline
\end{tabular}

${ }^{4}[\mathrm{X} / \mathrm{Y}]=\log (\mathrm{X} / \mathrm{Y})-\log (\mathrm{X} / \mathrm{Y})_{\odot}$. Solar abundances are from Asplund et al. (2005).

${ }^{5} t_{\mathrm{e}}=10^{-4} T_{\mathrm{e}}$. 